\title{
21. QUANTITATIVE DISTRIBUTION PATTERNS AND BIOMAGNETOSTRATIGRAPHY OF MIDDLE AND LATE MIOCENE CALCAREOUS NANNOFOSSILS FROM EQUATORIAL INDIAN AND PACIFIC OCEANS (LEGS 115, 130, AND 138) ${ }^{\prime}$
}

\author{
Isabella Raffi, ${ }^{2}$ Domenico Rio, ${ }^{3}$ Anna d'Atri, ${ }^{4}$ Eliana Fornaciari, ${ }^{3}$ and Silvana Rocchetti ${ }^{3}$
}

\begin{abstract}
Selected calcareous nannofossils were investigated by means of quantitative methods in middle and upper Miocene sediments from the tropical Indian Ocean (ODP Leg 115) and equatorial Pacific Ocean (DSDP Leg 85, ODP Legs 130 and 138). Our goal was to test the reliability of the classic biohorizons used in the standard zonations of Martini (1971) and Bukry (1973) and, possibly, to improve biostratigraphic resolution in the Miocene.

In a time interval of about $8 \mathrm{~m}$.y., from the last occurrence (LO) of $S$. heteromorphus $(\approx 13.6 \mathrm{Ma})$ to the LO of D. quinqueramus $(\approx 5.5 \mathrm{Ma})$, a total 37 events were investigated, using both the conventional and some additional markers proposed in the literature. At least 17 of these events proved to be distinct biostratigraphic correlation lines between the two considered areas. This integrated biostratigraphic framework increases the biostratigraphic resolution in the middle-upper Miocene interval (of the order of about $0.5 \mathrm{~m} . \mathrm{y})$.

All the investigated events were tied to the geomagnetic polarity time scale (GPTS) and compared to biomagnetostratigraphy from mid-latitude North Atlantic Site 94-608 (Olafsson, 1991; Gartner, 1992), thus obtaining further information about the biostratigraphic and biochronologic reliability of the investigated events and a significant improvement of the available nannofossil biomagnetostratigraphic model for the middle and late Miocene.
\end{abstract}

\section{INTRODUCTION}

Recently, considerable efforts have been dedicated to improving, by means of calcareous nannofossil events, the time resolution obtainable for the Miocene record, which is low when compared to those of the Pliocene and Pleistocene (Olafsson, 1989, 1991; Rio et al., 1990a; Fornaciari et al., 1990, 1993; Gartner, 1992). Furthermore, the purpose of these attempts was to test the nannofossil biostratigraphic reliability in the Miocene record, in terms of isochroneity of the bioevents and reproducibility over wide geographic areas.

Here, we expand the existing database for middle and late Miocene nannofossils by showing quantitative distribution patterns of selected species in sections from the equatorial Indian and Pacific oceans, recovered during DSDP Leg 85 and ODP Legs 115, 130, and 138 (Fig. 1 and Table 1). Quantitative distribution patterns are fundamental for evaluating reliability of the classic first occurrence (FO) and last occurrence (LO) events (Backman and Shackleton, 1983). In addition, detailed quantitative distribution patterns can provide supplementary events based on abundance fluctations (acme, absenceor "paracme"-intervals). These additional events can result in biostratigraphically useful data for regional and long-distance correlations in so far as they reflect regional paleoceanographic events and/or global evolutionary turnovers that do not result in extinctions. Quantitative distribution patterns, as well, are important for gaining insights into the paleoecology and biogeography of calcareous nannofossils and for overcoming many traditional problems of biostratigraphy, such as noises in the stratigraphic record (reworking, etc.)

\footnotetext{
${ }^{1}$ Pisias, N.G., Mayer, L.A., Janecek, T.R., Palmer-Julson, A., and van Andel, T.H. (Eds.), 1995. Proc. ODP, Sci. Results, 138: College Station, TX (Ocean Drilling Program). ${ }^{2}$ Università di Parma, Istituto di Geologia, Italia. Present address: Università degli Studi "G. D'Annuzio," Chieti-Facoltà di Scienze Matematiche, Fisiche e Naturali.

${ }^{3}$ Universita di Padova, Dipartimento di Geologia, Paleontologia e Geofisica, Via Giotto 1, 35137 Padova, Italia.

${ }^{4}$ Università di Torino, Dipartimento di Scienze della Terra, Via Accademia delle Scienze 5, 10123 Torino, Italia.
}

and inconsistencies in data-collecting methodologies (see discussion in Backman and Shackleton, 1983; Rio et al., 1990b).

\section{OBJECTIVES AND STRATEGY}

As stated above, our main objectives were to test the reliability of calcareous nannofossil biohorizons (biostratigraphic events) and to improve biostratigraphic resolution. To make our objectives and conclusions more clear, we found it necessary to state our concept of biostratigraphic reliability, a much discussed topic in the past (i.e., Gradstein, 1985; Hill and Thierstein, 1989; Rio et al., 1990b; Bralower et al.,1989). In our concept, a biohorizon is considered to be reliable when it is easily reproducible among the different researchers and can be consistently correlated among distant and/or different facies/ sections, maintaining its position relative to other biohorizons. This last property, recently named "ranking" by Gradstein (1985), is simply the scientific paradigm of biostratigraphy (the principle of faunal succession) on which William Smith at the end of the 18th century founded biostratigraphy (Prothero, 1990). Reproducibility of a biohorizon is function of (1) the clear taxonomy of the index species; (2) the mode of occurrence (subtle, abrupt, etc.) of the change in distribution pattern of the index species one chooses as biohorizon.

Therefore, we will rank biostratigraphic reliability of an index species by evaluating the following:

1. Unambiguous taxonomy;

2. Mode of occurrence of the event by visual inspection of the abundance pattern ("morphology" of the event) of the index species;

3. Consistency of the relative position (ranking) with respect to other biohorizons in distant sections; and

4. Position of the events vs. the available chronomagnetostratigraphy.

\section{MATERIAL AND METHODS}

The sections included in this study are located in Figure 1 and listed in Table 1, where pertinent references for location and background information on the 10 sites investigated and on the sites considered for comparison are reported. Except for DSDP Site 608, 
Table 1. Summary of cores considered in this study.

\begin{tabular}{|c|c|c|c|c|c|c|}
\hline Leg & Site/hole & $\begin{array}{l}\text { Location } \\
\text { (lat, long) }\end{array}$ & $\begin{array}{l}\text { Water } \\
\text { depth } \\
\text { (m) }\end{array}$ & $\begin{array}{l}\text { General } \\
\text { lithology }\end{array}$ & Magnetics & References \\
\hline DSDP 82 & 558 & $\begin{array}{l}\text { Western North Atlantic Ocean } \\
\left(37^{\circ} 46.2^{\prime} \mathrm{N}, 37^{\circ} 20.61^{\prime} \mathrm{W}\right)\end{array}$ & 3754 & Nannofossil ooze & Khan et al. (1985) & $\begin{array}{l}\text { Bukry (1985); Miller et al. (1985); } \\
\text { Parker (1985) }\end{array}$ \\
\hline DSDP 82 & 563 & $\begin{array}{l}\text { Western North Atlantic Ocean } \\
\left(33^{\circ} 38.53^{\prime} \mathrm{N}, 43^{\circ} 46.04^{\prime} \mathrm{W}\right)\end{array}$ & 3786 & $\begin{array}{l}\text { Foraminiferal nannofossil ooze } \\
\text { and chalk }\end{array}$ & Khan et al. (1985) & $\begin{array}{l}\text { Bukry (1985); Miller et al. (1985); } \\
\text { Parker (1985) }\end{array}$ \\
\hline DSDP 94 & 608 & $\begin{array}{c}\text { Eastern North Altantic Ocean } \\
\left(42^{\circ} 50.20^{\prime} \mathrm{N}, 23^{\circ} 05.25^{\prime} \mathrm{W}\right)\end{array}$ & 3526 & $\begin{array}{l}\text { Foraminifer and nannofossil ooze } \\
\text { and chalk }\end{array}$ & Clement and Robinson (1987) & $\begin{array}{l}\text { Takayama and Sato (1987); } \\
\text { Olafsson (1991); Gartner (1992) }\end{array}$ \\
\hline DSDP 85 & 574 & $\begin{array}{l}\text { Central equatorial Pacific Ocean } \\
\left(4^{\circ} 12.52^{\prime} \mathrm{N}, 133^{\circ} 19.81^{\prime} \mathrm{W}\right)\end{array}$ & 4561 & Calcareous ooze chalk & - & Pujos (1985); Olafsson (1989) \\
\hline DSDP 85 & 575 & $\begin{array}{l}\text { Central equatorial Pacific Ocean } \\
\left(5^{\circ} 51.00^{\prime} \mathrm{N}, 135^{\circ} 02.16^{\prime} \mathrm{W}\right)\end{array}$ & 4536 & Siliceous and nannofossil ooze & - & Pujos (1985); present study \\
\hline ODP 130 & 806 & $\begin{array}{l}\text { Western equatorial Pacific Ocean } \\
\left(0^{\circ} 19.11^{\prime} \mathrm{N}, 159^{\circ} 21.68^{\prime} \mathrm{E}\right)\end{array}$ & 2520.7 & Foraminiferal nannofossil chalk & - & $\begin{array}{l}\text { Fornaciari et al. (1993); present } \\
\text { study }\end{array}$ \\
\hline ODP 138 & 844 & $\begin{array}{l}\text { Eastern equatorial Pacific Ocean } \\
\left(7^{\circ} 55.28^{\prime} \mathrm{N}, 90^{\circ} 28.85^{\prime} \mathrm{W}\right)\end{array}$ & 3425.0 & $\begin{array}{l}\text { Clay-rich biogenic, silica-rich } \\
\text { ooze + nannofossil ooze }\end{array}$ & $\begin{array}{l}\text { Mayer, Pisias, Janecek, et al. (1992), } \\
\text { Schneider (this volume) }\end{array}$ & $\begin{array}{l}\text { Raffi and Flores (this volume); } \\
\text { present study }\end{array}$ \\
\hline ODP 138 & 848 & $\begin{array}{c}\text { Eastem equatorial Pacific Ocean } \\
\left(2^{\circ} 59.63^{\prime} \mathrm{S}, 110^{\circ} 28.79^{\prime} \mathrm{W}\right)\end{array}$ & 3867.3 & Foraminiferal nannofossil ooze & $\begin{array}{l}\text { Mayer, Pisias, Janecek, et al. (1992), } \\
\text { Schneider et al. (this volume) }\end{array}$ & $\begin{array}{l}\text { Raffi and Flores (this volume); } \\
\text { present study }\end{array}$ \\
\hline ODP 138 & 853 & $\begin{array}{l}\text { Eastern equatorial Pacific Ocean } \\
\left(7^{\circ} 12.66^{\prime} \mathrm{N}, 109^{\circ} 45.08^{\prime} \mathrm{W}\right)\end{array}$ & 3727.2 & Clayey nannofossil ooze & Mayer, Pisias, Janecek, et al. (1992) & \\
\hline ODP 115 & 709 & $\begin{array}{l}\text { Western tropical Indian Ocean } \\
\left(3^{\circ} 54.72^{\prime} \mathrm{S}, 60^{\circ} 33.16^{\prime} \mathrm{E}\right)\end{array}$ & 3046.9 & Nannofossil ooze & - & $\begin{array}{l}\text { Fornaciari ct al. (1990); } \\
\text { Rio et al. (1990); present study }\end{array}$ \\
\hline ODP 115 & 710 & $\begin{array}{l}\text { Western tropical Indian Ocean } \\
\left(4^{\circ} 18.69^{\prime} \mathrm{S}, 60^{\circ} 48.76^{\prime} \mathrm{E}\right)\end{array}$ & 3822.5 & Clay-bearing nannofossil ooze & Schneider and Kent (1990) & $\begin{array}{l}\text { Backman et al. (1990); } \\
\text { Rio et al. (1990); present study }\end{array}$ \\
\hline ODP 115 & 711 & $\begin{array}{l}\text { Western tropical Indian Ocean } \\
\left(2^{\circ} 44.46^{\prime} \mathrm{S}, 61^{\circ} 09.75^{\prime} \mathrm{E}\right)\end{array}$ & 4438.7 & Clay and clayey nannofossil ooze & Schneider and Kent (1990) & Rio et al. (1990); present study \\
\hline ODP 115 & 714 & $\begin{array}{l}\text { Western tropical Indian Ocean } \\
\left(5^{\circ} 03.69^{\prime} \mathrm{N}, 73^{\circ} 46.98^{\prime} \mathrm{E}\right)\end{array}$ & 2042.0 & Foraminiferal nannofossil ooze & - & $\begin{array}{l}\text { Fomaciari et al. (1990); Rio et al. } \\
\text { (1990); present study }\end{array}$ \\
\hline
\end{tabular}

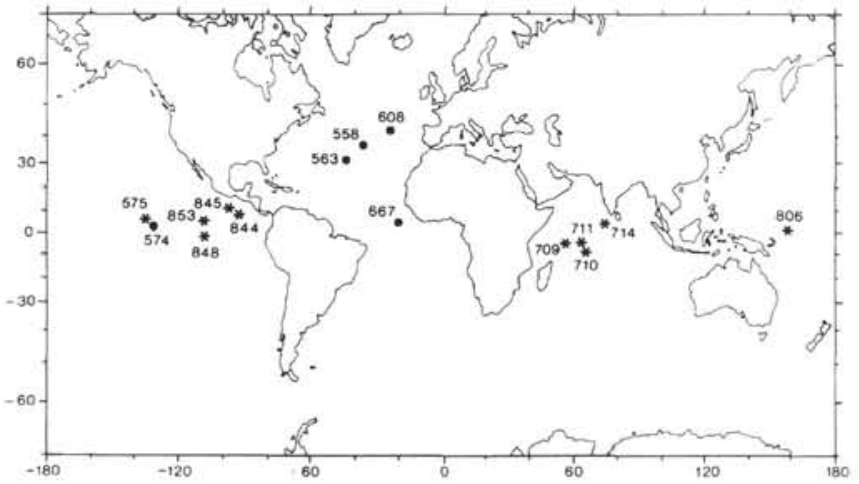

Figure 1. Location of DSDP and ODP Sites studied $\left(^{*}\right)$ and considered for reference $(\bullet)$.

all are located in low-latitude areas, but represent vastly different watermasses. The temporal extension of the various sections is summarized in Figure 2, where the availability of magnetostratigraphic records is evidenced.

Light microscope techniques were used for examining smear slides, which were made directly from core samples, using standard methods. Quantitative data were collected according to three methods:

1. Counting the index species relative to the total assemblage.

2. Counting the index species relative to a prefixed number of taxonomically related forms (i.e., species of discoasterids relative to 200 discoasterids, etc.).

3. Counting the number of specimens of the index species per unit area of the slide.
Backman and Shackleton (1983) and Rio et al. (1990b) discussed these three methods at length, indicating their respective advantages as well as their limits of applicability.

Method 1 was applied for evaluating abundances of Mynilitha convallis, and of all the index species reported at Site 806. Method 2 was applied for evaluating abundances of discoasterid species, Calcidiscus premacintyrei and $C$. macintyrei $(\geq 11 \mu \mathrm{m})$, Sphenolithus heteromorphus. Method 3 was applied for obtaining distribution patterns of ceratolithids and triquetrorhabdulid species and Coronocyclus nitescens. As regards Reticulofenestra pseudoumbilicus $(>7 \mu \mathrm{m})$ and $C y$ clicargolithus floridanus, different counting methods were applied in the different sequences. $R$. pseudoumbilicus was counted vs. all the other nannofossils at Sites 575 (Fig. 3), 714 (Fig. 4), and 806 (Fig. 5), and vs. C. floridanus at Site 845 (Fig. 6). Except in this latter site, $C$. floridanus was counted vs. all the nannofossils at Sites 575 (Fig. 3) and 806 (Fig. 5) and per unit area at Site 714 (Fig. 4). Note that the different methods provide comparable biostratigraphic signals.

As regards the magnetostratigraphic records reported in the investigated sequences, data are from Schneider and Kent (1990) for ODP Leg 115 sequences (Figs. 10 and 14), and from site chapters in Mayer, Pisias, and Janecek, et al. (1992) and Schneider (this volume) for ODP Leg 138 sequences (Figs. 6, 11-13, 15). We refer to the paleomagnetic time scale of Cande and Kent (1992), combined with the time scale developed for Leg 138 sites (Shackleton et al., this volume) (Fig. 17, back pocket).

\section{REMARKS ON TAXONOMY}

Calcareous nannofossil species considered in this study are listed in the Appendix. Most of these are referenced in Perch-Nielsen (1985). Consistency in taxonomic concepts is a key factor in biostratigraphy, and the use of different taxonomic concepts (expecially when dealing with intermediate morphologies of evolving lineages) explains much of the differences in biostratigraphic ranges found in literature. We have made clear the adopted taxonomic concepts in previous papers 


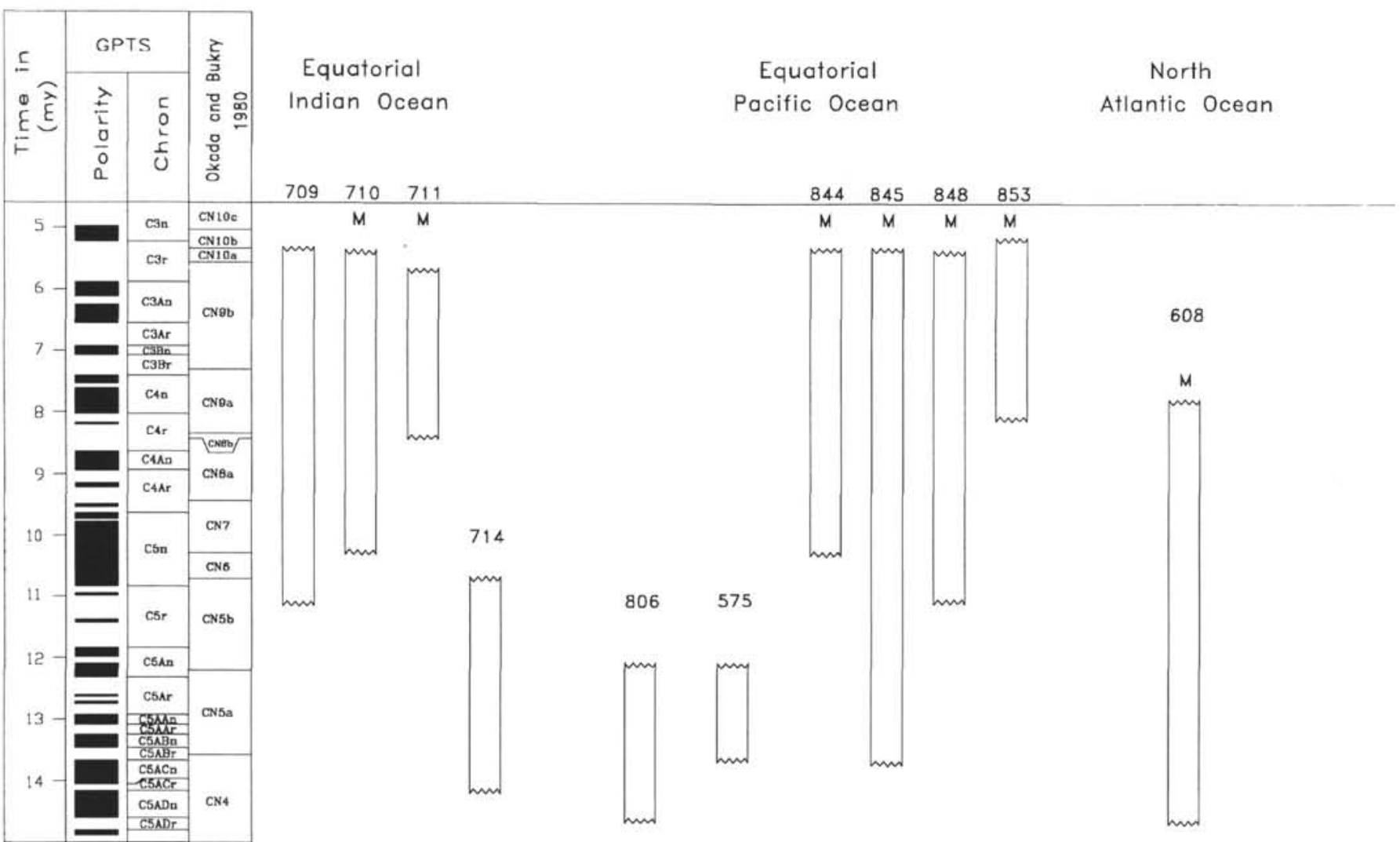

Figure 2. Position relative to integrated biomagnetostratigraphy of the investigated sections. " $M$ " denotes sections with magnetostratigraphy.

(Rio et al., 1990a; Fornaciari et al., 1990; Raffi and Flores, this volume), to which the reader is referred. When necessary, we will reiterate the adopted criteria in some of the index species determination, commenting on a single biohorizon.

\section{RESULTS}

We established by different counting methods the distribution patterns of 29 middle and late Miocene index calcareous nannofossils, shown in Figures 3 to 16. Besides the distribution of index nannofossils, in some sequences we report also the distribution patterns of additional species, such as Discoaster musicus, D. aff. calcaris, and Discoaster sp. 2, which characterize the assemblages at different stratigraphic intervals. Specifically, $D$. aff. calcaris is a discoasterid observed both in tropical Indian and equatorial Pacific oceans within Zone CN5 (see Rio et al., 1990a, for description). Discoaster sp. 2 is a large discoasterid similar to Discoaster brouweri, consistently recorded within Subzone CN9b, in both low-latitude areas (Rio et al., $1990 \mathrm{a}$; Raffi and Flores, this volume). Moreover, as regards discoasterids, we did not report quantitative distributions either of long-ranging species, which do not provide significative biostratigraphic signal (such as D. brouweri and D. variabilis), or of index species found as rare and scattered. Among this latter group, we included Discoaster braarudii, which does not result in a reliable biostratigraphic marker in the middle Miocene, as suggested by Gartner (1992). Both in tropical Indian Ocean sequences (ODP Leg 115) and equatorial Pacific Ocean sequences (ODPLeg 138), D. braarudii is rare, discontinuously distributed in Zones CN6 and CN7 (NN8 and NN9) and does not have any biostratigraphic utility.

On the basis of the established distribution patterns, their long distance correlations, and their calibration to the available magnetostratigraphy (Fig. 17, back pocket), we discuss in the following sections the reliability of 37 biohorizons, shown in Table 2 and numbered
Table 2. Summary of biohorizons considered in this study.

\begin{tabular}{|c|c|}
\hline 37 & Discoaster quinqueramus LO \\
\hline 36 & Amaurolithus amplificus LO \\
\hline 35 & Amaurolithus amplificus FO \\
\hline 34 & Amaurolithus primus FO \\
\hline 33 & Discoaster surculus FCO \\
\hline 32 & Discoaster berggrenii $\mathrm{FO}$ \\
\hline 31 & Discoaster pentaradiatus FO \\
\hline 30 & Discoaster neorectus FO \\
\hline 29 & Discoaster loeblichii FO \\
\hline 28 & Discoaster bollii LO \\
\hline 27 & Mynilitha convallis FO \\
\hline 26 & Discoaster hamatus LO \\
\hline 25 & Discoaster neohamatus FO \\
\hline 24 & Discoaster prepentaradiatus FO \\
\hline 23 & Catinaster calyculus LO \\
\hline 22 & Catinaster coalitus LO \\
\hline 21 & Discoaster hamatus FO \\
\hline 20 & Catinaster calyculus FO \\
\hline 19 & Coccolithus miopelagicus LO \\
\hline 18 & Discoaster bellus group FO \\
\hline 17 & Discoaster exilis LCO \\
\hline 16 & Discoaster calcaris FO \\
\hline 15 & Catinaster coalitus FO \\
\hline 14 & Discoaster kugleri LO \\
\hline 13 & Discoaster kugleri LCO \\
\hline 12 & Discoaster bollii FO \\
\hline 11 & Discoaster kugleri FCO \\
\hline 10 & Discoaster kugleri FO \\
\hline 9 & Calcidiscus macintyrei $\mathrm{FO}$ \\
\hline 8 & Coronocyclus nitescens LO \\
\hline 7 & Triquetrorhabdulus serratus LO \\
\hline 6 & Calcidiscus premacintyrei LCO \\
\hline 5 & Triquetrorhabdulus rugosus FCO \\
\hline 4 & Discoaster signus LO \\
\hline 3 & Cyclicargolithus floridanus LCO \\
\hline 2 & Reticulofenestra pseudoumbilicus FO \\
\hline 1 & Sphenolithus heteromorphus LO \\
\hline
\end{tabular}

Notes: $\mathrm{LO}=$ last occurrence; $\mathrm{FO}=$ first ocurrance; $\mathrm{LCO}=$ last common occurrence. 


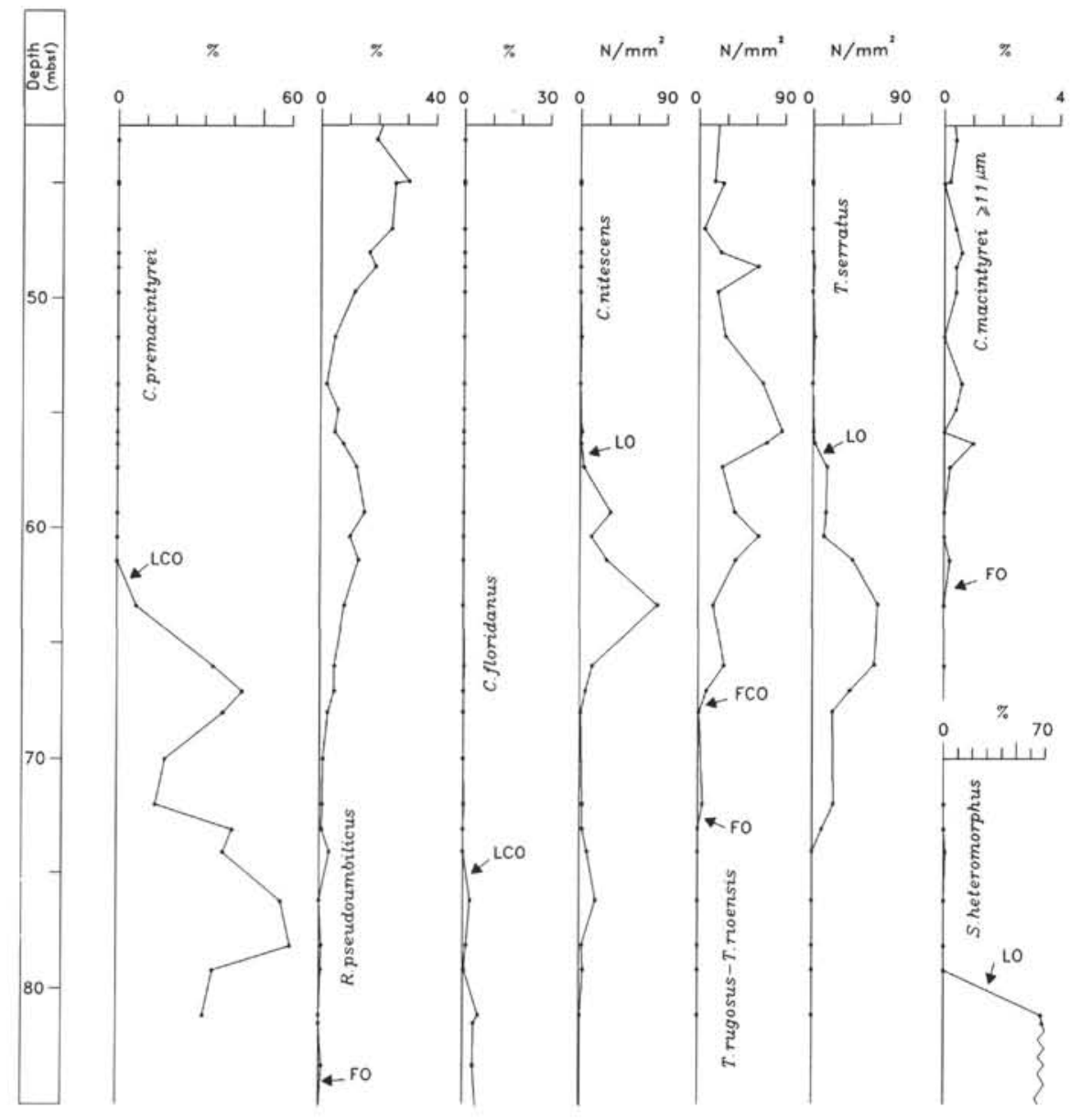

Figure 3. Abundance patterns of middle Miocene selected calcareous nannofossils at Hole 575B. (mbsf) $=$ meters below seafloor. $\mathrm{N} / \mathrm{mm}^{2}=$ number of specimens per square millimeter; $\mathrm{FO}=$ first occurrence; $\mathrm{LO}=$ last occurrence $; \mathrm{FCO}=$ first common and/or continuous occurrence $\mathrm{LCO}=$ last common and/or continuous occurrence.

Table 3. Position of calcareous nannofossil events at Hole 575B.

\begin{tabular}{lll}
\hline \multicolumn{1}{c}{ Event } & \multicolumn{1}{c}{$\begin{array}{c}\text { Core, section } \\
(\mathrm{cm})\end{array}$} & $\begin{array}{c}\text { Depth } \\
(\mathrm{mbsf})\end{array}$ \\
\hline $\begin{array}{l}\text { D. hamatus FO } \\
\text { C. miopelagicus } \mathrm{LO}\end{array}$ & $5 \mathrm{H}-3,115 / 5 \mathrm{H}-2,28$ & $43.15-40.78$ \\
C. nitescens $\mathrm{LO}$ & $7 \mathrm{H}-3,115 / 5 \mathrm{H}-2,28$ & $43.15-40.78$ \\
T. serratus $\mathrm{LO}$ & $7 \mathrm{H}-2,49 / 7 \mathrm{H}-1,95$ & $57.39-56.35$ \\
C. macintyrei $\mathrm{FO}$ & $7 \mathrm{H}-6,52 / 7 \mathrm{H}-5,5$ & $57.39-56.35$ \\
C. premacintyrei $\mathrm{LCO}$ & $7 \mathrm{H}-6,52 / 7 \mathrm{H}-5,5$ & $63.42-61.45$ \\
T. rugosus $\mathrm{FCO}$ & $8 \mathrm{H}-3,31 / 8 \mathrm{H}-2,90$ & $63.42-61.45$ \\
C. floridanus $\mathrm{LCO}$ & $9 \mathrm{H}-2,93 / 9 \mathrm{H}-1,32$ & $76.23-74.12$ \\
S. heteromorphus $\mathrm{LO}$ & $9 \mathrm{H}-5,137 / 9 \mathrm{H}-4,92$ & $81.17-79.22$ \\
R. pseudoumbilicus $\mathrm{FO}$ & $10 \mathrm{H}-3,108 / 10 \mathrm{H}-2,47$ & $85.48-83.87$ \\
\hline
\end{tabular}

Notes: $\mathrm{FO}=$ first occurrence $; \mathrm{LO}=$ last occurrence; $\mathrm{FCO}=$ first common and continuous occurrence; $\mathrm{LCO}=$ last common and continuous occurrence.

in stratigraphic order. The stratigraphic position in the single sections of these biohorizons is summarized in Tables 3 to 13 .

\section{LO of Sphenolithus heteromorphus (1)}

The LO of the easily identified Sphenolithus heteromorphus (definition of the top of Zones CN4 and NN5) appears as one of the most easily determined and correlatable event in the investigated sections (Figs. 3, 5, and 6), as is generally acknowledged in the literature (i.e., Olafsson 1989, 1991). Problems with the LO of S. heteromorphus arise with calibration to the GPTS. In fact, Berggren et al. (1985) associated
Table 4. Position of calcareous nannofossil events at Hole 714A.

\begin{tabular}{|c|c|c|}
\hline Event & $\begin{array}{l}\text { Core, section } \\
\text { (cm) }\end{array}$ & $\begin{array}{l}\text { Depth } \\
\text { (mbsf) }\end{array}$ \\
\hline M. convallis $\mathrm{FO}$ & $4 \mathrm{H}-7,140 / 4 \mathrm{H}-7,75$ & $31.7-31.05$ \\
\hline D. hamatus LO & $5 \mathrm{H}-1,30 / 4 \mathrm{H}-7,140$ & $32.0-31.7$ \\
\hline D. pentaradiatus FO & $5 \mathrm{H}-1,140 / 5 \mathrm{H}-1,30$ & $33.1-32.0$ \\
\hline D. bollii LO & $5 \mathrm{H}-4,30 / 5 \mathrm{H}-3,140$ & $36.5-36.1$ \\
\hline D. prepentaradiatus FO & $6 \mathrm{H}-1,30 / 5 \mathrm{H}-7,30$ & $41.7-41.0$ \\
\hline C. coalitus $\mathrm{LO}$ & $7 \mathrm{H}-1,30 / 6 \mathrm{H}-7-30$ & $51.3-50.7$ \\
\hline D. neohamatus $\mathrm{FO}$ & $7 \mathrm{H}-2,140 / 7 \mathrm{H}-2,30$ & $53.9-52.8$ \\
\hline D. hamatus FO & $7 \mathrm{H}-4,30 / 7 \mathrm{H}-4,10$ & $55.8-55.6$ \\
\hline D. bellus group FO & $7 \mathrm{H}-4,30 / 7 \mathrm{H}-4,10$ & $55.8-55.6$ \\
\hline C. miopelagicus LO & $7 \mathrm{H}-4,75 / 7 \mathrm{H}-6,10$ & $58.6-56.25$ \\
\hline C. calyculus $\mathrm{FO}$ & Not reliable & \\
\hline D. exilis LCO & $7 \mathrm{H}-7,30 / 7 \mathrm{H}-6,140$ & $60.30-59.9$ \\
\hline D. kugleri LO & $8 \mathrm{H}-1,75 / 7 \mathrm{H}-7,30$ & $61.35-60.3$ \\
\hline D. calcaris FO & $8 \mathrm{H}-1,140 / 7 \mathrm{H}-7,30$ & $62.0-60.3$ \\
\hline C. coalitus FO & $8 \mathrm{H}-1,140 / 8 \mathrm{H}-1,75$ & $62.0-61.35$ \\
\hline D. bollii FO & $8 \mathrm{H}-4,75 / 8 \mathrm{H}-3,140$ & $65.9-65.0$ \\
\hline D. kugleri FO & Not reliable & \\
\hline C. nitescens LO & $10 \mathrm{H}-1,30 / 9 \mathrm{H}-5,30$ & $80.1-76.5$ \\
\hline T. serratus LO & $10 \mathrm{H}-1,30 / 9 \mathrm{H}-5,30$ & $80.1-76.5$ \\
\hline R. pseudoumbilicus FO & $10 \mathrm{H}-6,75 / 10 \mathrm{H}-5,130$ & $88.05-87.1$ \\
\hline$S$. heteromorphus LO & $10 \mathrm{H}-6,75 / 10 \mathrm{~h}-5,130$ & $88.05-87.1$ \\
\hline T. rugosus FCO & $10 \mathrm{H}-6,140 / 10 \mathrm{H}-6,30$ & $88.7-87.6$ \\
\hline C. floridanus LCO & $10 \mathrm{H}-6,140 / 10 \mathrm{H}-6,30$ & $88.7-87.6$ \\
\hline
\end{tabular}

Notes: As specified in Table 3. 


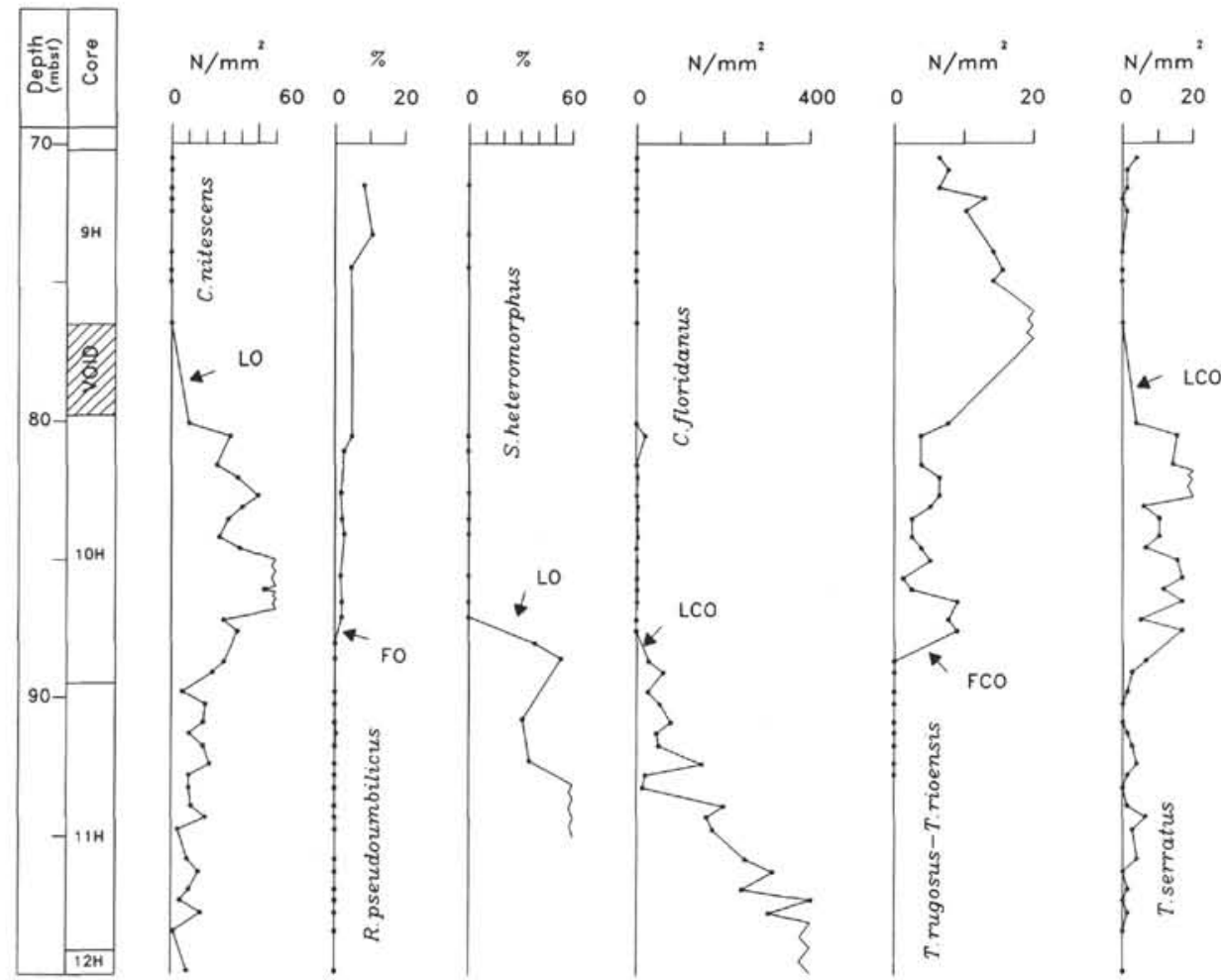

Figure 4. Abundance patterns of middle Miocene selected calcareous nannofossils at Hole 714A. $(\mathrm{mbsf})=$ meters below seafloor. Notation as specified in Figure 3.

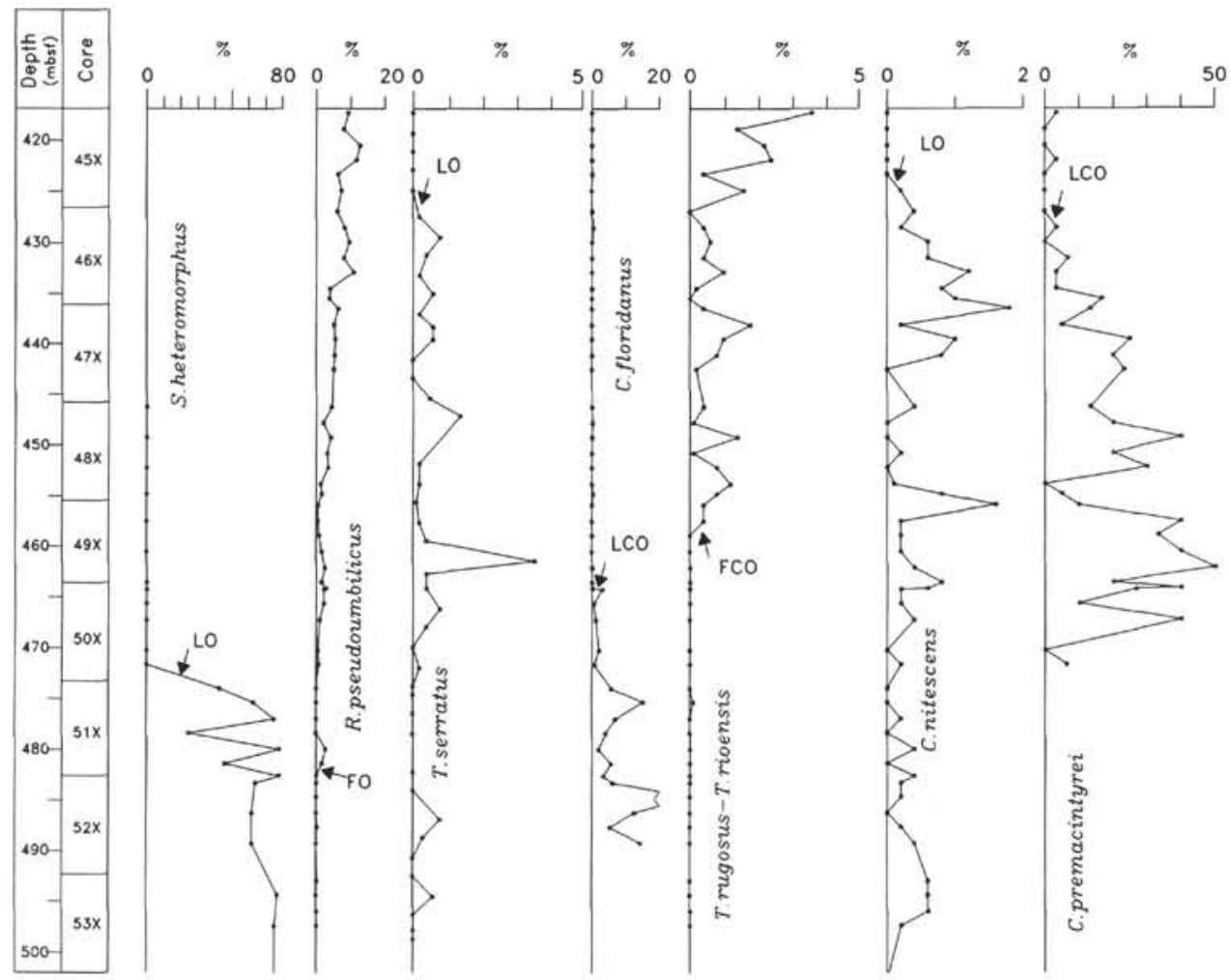

Figure 5. Abundance patterns of middle Miocene selected calcareous nannofossils at Hole 806B. (mbsf) $=$ meters below seafloor. Notation as specified in Figure 3. 


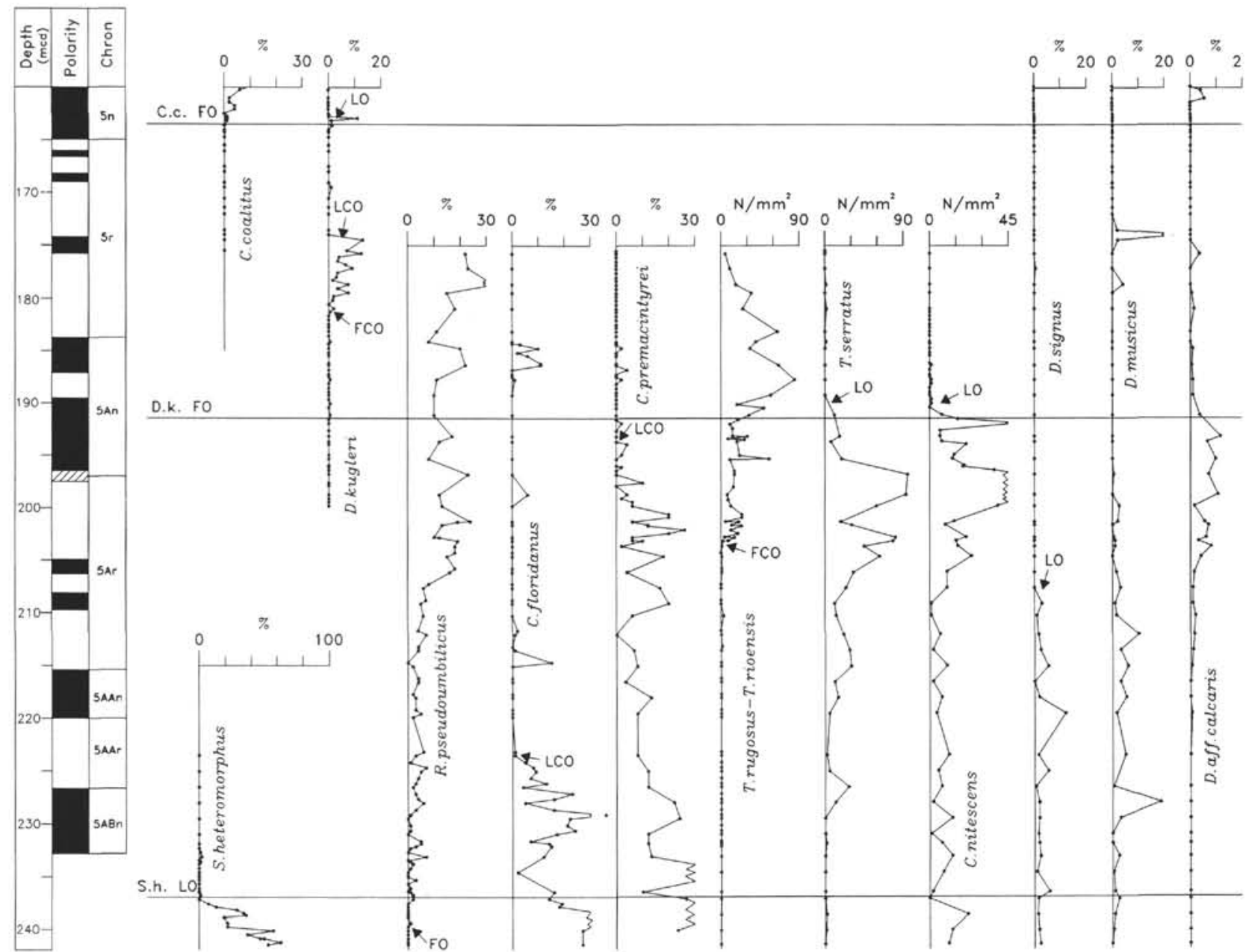

Figure 6. Abundance patterns of middle Miocene selected calcareous nannofossils at Site 845. $(\mathrm{mcd})=$ meters composite depth. Notation as specified in Figure 3. C.c. $=$ Catinaster coalitus; D.k. $=$ Discoaster kugleri; S.h. $=$ Sphenolithus heteromorphus . Magnetostratigraphy from Schneider (this volume).

Table 5. Position of calcareous nannofossil events at Hole 806B.

\begin{tabular}{lll}
\hline \multicolumn{1}{c}{ Event } & \multicolumn{1}{c}{$\begin{array}{c}\text { Core, section } \\
(\mathrm{cm})\end{array}$} & \multicolumn{1}{c}{$\begin{array}{c}\text { Depth } \\
(\mathrm{mbs})\end{array}$} \\
\hline $\begin{array}{l}\text { D. kugleri FO } \\
\text { C. nitescens LO }\end{array}$ & $\begin{array}{l}\text { Not detected } \\
45 X-6,60 / 45 X-5,50\end{array}$ & $425.0-423.4$ \\
C. macintyrei FO & Not detected & \\
T. serratus LO & $45 \mathrm{X}-6,60 / 46 \mathrm{X}-1,50$ & $427.0-425.0$ \\
C. premacintyrei LCO & $46 \mathrm{X}-2,60 / 46 \mathrm{X}-1,50$ & $428.6-427.0$ \\
T. rugosus FCO & $48 \mathrm{X}-3,50 / 49 \mathrm{X}-2,60$ & $459.0-457.6$ \\
C. floridanus LCO & $50 \mathrm{X}-1,70 / 49 \mathrm{X}-7,30$ & $464.3-464.2$ \\
S. heteromorphus LO & $51 \mathrm{X}-1,70 / 50 \mathrm{X}-6,60$ & $474.0-471.7$ \\
R. pseudoumbilicus FO & $51 \mathrm{X}-6,60 / 51 \mathrm{X}-7,30$ & $482.6-481.4$ \\
\hline
\end{tabular}

Notes: As specified in Table 3.

this important event with Chron 5ADn, considering the results of Miller et al. (1985) at DSDP Sites 558 and 563, whereas Backman et al. (1990) and Olafsson (1991) associated it to Chron $5 \mathrm{ABr}$ on the basis of results from DSDP Site 608. About $1 \mathrm{~m} . \mathrm{y}$. of diachroneity is inferred when accepting these data. However, note that recently, Miller et al. (1991) correlated, via oxygen isotope stratigraphy, Sites 563 and 608 , and reinterpreted the magnetostratigraphic record of the former succession (compare Fig. 1 in Miller et al., 1985, with Fig. 4 in Miller et al., 1991). At both sites, $S$. heteromorphus LO is almost coincident with a prominent stable oxygen isotope minimum in benthic foraminifers (Mi3), which in the new interpretation of Miller et al. (1991), is associated in both sites with Chron $5 \mathrm{ABr}$. A similar calibration can be inferred also at Site 521 (Hsü et al., 1984) and at Indian Ocean Site 710 (Rio et al., 1990a), where the LO of S. heteromorphus clearly postdates Chron 5AD time.

No reliable magnetostratigraphy is available in the sequences of Leg 138 sediments in the interval around the LO of $S$. heteromorphus. However, extrapolating sediment accumulation rates at Site 845 , an age corresponding to the top Chron $5 \mathrm{ACn}$ - base Chron $5 \mathrm{ABr}$ timeis obtained (Raffi and Flores, this volume).

We consider the $S$. heteromorphus LO an excellent biostratigraphic event, which has probably a high chronostratigraphic correlation potential between low- and mid-latitude areas (Fig. 17, back pocket).

\section{FO of Reticulofenestra pseudoumbilicus (2)}

For distinguishing Reticulofenestra pseudoumbilicus, we considered reticulofenestrids larger than $7 \mu \mathrm{m}$, following the taxonomic concepts of Raffi and Rio (1979) and Backman and Shackleton (1983). These reticulofenestrids provide a biostratigraphically and biochronologically reliable event in the Pliocene, when they became extinct in the upper part of Chron 2Ar (late Gilbert) (Backman and Shackleton, 1983; Rio et al. 1990a, 1990b; Raffi and Flores, this 


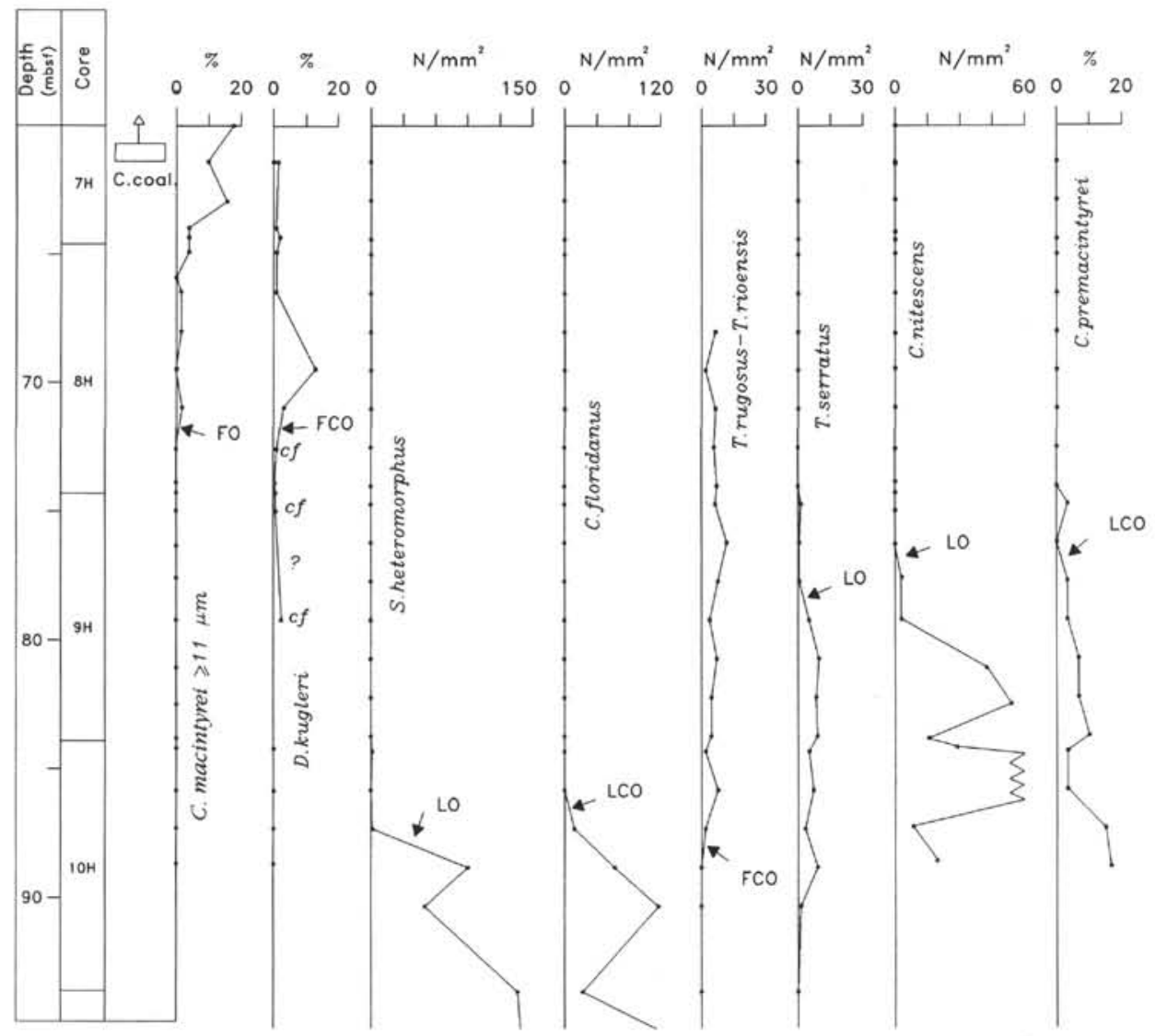

Figure 7. Abundance patterns of middle Miocene selected calcareous nannofossils at Hole 714B. (mbsf) $=$ meters below seafloor. Notation as specified in Figure 3.

Table 6. Position of calcareous nannofossil events at Site 845.

\begin{tabular}{|c|c|c|}
\hline Event & $\begin{array}{l}\text { Core, section } \\
\text { (cm) }\end{array}$ & $\begin{array}{l}\text { Depth } \\
\text { (mcd) }\end{array}$ \\
\hline D. quinqueramus LO & $845 \mathrm{~A}-8 \mathrm{H}-7,12 / 8 \mathrm{H}-6,120$ & $80.02-79.60$ \\
\hline A. amplificus LO & Not reliable & \\
\hline A. amplificus $\mathrm{FO}$ & $845 \mathrm{~A}-10 \mathrm{H}-4,13 / 10 \mathrm{H}-3,120$ & $96.71-96.28$ \\
\hline A. primus $\mathrm{FO}$ & $845 \mathrm{~A}-11 \mathrm{H}-2,120 / 11 \mathrm{H}-2,32$ & $105.56-104.68$ \\
\hline D. surculus FCO & $845 \mathrm{~A}-12 \mathrm{H}-1,120 / 12 \mathrm{H}-1,42$ & $115.13-114.35$ \\
\hline D. berggrenii $\mathrm{FO}$ & $845 \mathrm{~B}-12 \mathrm{H}-5,150 / 845 \mathrm{~A} 13 \mathrm{H}-1,145$ & $126.58-126.16$ \\
\hline D. pentaradiatus $\mathrm{FO}$ & Not reliable & \\
\hline D. loeblichii LO & Not reliable & \\
\hline D. loeblichii FO & Not reliable & \\
\hline D. bollii LO & $845 \mathrm{~A} 15 \mathrm{H}-5,98 / 15 \mathrm{H}-5,70$ & $153.94-153.66$ \\
\hline D. hamatus LO & Not reliable & \\
\hline D. neohamatus FO & Not detected & \\
\hline D. exilis LCO & $845 \mathrm{~B} 15 \mathrm{H}-2,100 / 15 \mathrm{H}-2,60$ & $156.9-156.54$ \\
\hline D. hamatus FO & $845 \mathrm{~B} 15 \mathrm{H}-2,145 / 15 \mathrm{H}-2,120$ & $57.35-157.1$ \\
\hline C. calyculus FO & $845 \mathrm{~A} 16 \mathrm{H}-1,42 / 845 \mathrm{~B} 15 \mathrm{H}-3,130$ & $158.75-158.7$ \\
\hline C. miopelagicus LO & $845 \mathrm{~B} 15 \mathrm{H}-4,40 / 15 \mathrm{H}-4,20$ & $159.3-159.1$ \\
\hline D. calcaris FO & $845 \mathrm{~A} 16 \mathrm{H}-2,42 / 845 \mathrm{~B} 15 \mathrm{H}-4,100$ & $160.25-159.9$ \\
\hline D. bellus group FO & $845 \mathrm{~A} 16 \mathrm{H}-2,120 / 16 \mathrm{H}-2,42$ & $161.03-160.25$ \\
\hline D. kugleri LO & $845 \mathrm{~A} 16 \mathrm{H}-4,11 / 16 \mathrm{H}-3,143$ & $162.94-162.76$ \\
\hline C. coalitus $\mathrm{FO}$ & $845 \mathrm{~A} 16 \mathrm{H}-4,78 / 16 \mathrm{H}-4,32$ & $163.61-163.15$ \\
\hline D. surculus FO & $845 \mathrm{~A} 16 \mathrm{H}-4,143 / 16 \mathrm{H}-4,120$ & $164.26-164.03$ \\
\hline D. kugleri LCO & $845 \mathrm{~B} 16 \mathrm{H}-7,20 / 16 \mathrm{H}-6,120$ & $174.0-173.56$ \\
\hline D. bollii FO & 845B $17 \mathrm{H}-2,79 / 17 \mathrm{H}-1,90$ & $177.19-175.8$ \\
\hline D. kugleri FCO & $845 \mathrm{~B} 17 \mathrm{H}-5,42 / 17 \mathrm{H}-5,10$ & $181.32-181$ \\
\hline D. kugleri FO & $845 \mathrm{~B} 18 \mathrm{H}-5,120 / 845 \mathrm{~B} 18 \mathrm{H}-5,78$ & $191.6-191.18$ \\
\hline C. nitescens LO & $845 \mathrm{~B} 18 \mathrm{H}-5,78 / 845 \mathrm{~B} 18 \mathrm{H}-5,10$ & $191.18-190.5$ \\
\hline$T$, serratus LO & $845 \mathrm{~B} 18 \mathrm{H}-5,78 / 18 \mathrm{H}-4,40$ & $191.18-189.3$ \\
\hline C. macintyrei $\mathrm{FO}$ & $845 \mathrm{~B} 18 \mathrm{H}-5,120 / 845 \mathrm{~B} 18 \mathrm{H}-5,78$ & $191.6-191.18$ \\
\hline C. premacintyrei LCO & $845 \mathrm{~B} 18 \mathrm{H}-7,50 / 18-\mathrm{H} 7,23$ & $194.0-193.73$ \\
\hline T. rugosus FCO & 845 B $19 \mathrm{H}-7,15 / 845$ А $20 \mathrm{H}-7.78$ & $203.74-203.21$ \\
\hline D. signus LO & $845 \mathrm{~A} 20 \mathrm{H}-6,78 / 20 \mathrm{H}-5,78$ & $209.21-207.71$ \\
\hline C. floridanus LCO & $845 \mathrm{~A} 22 \mathrm{H}-1,143 / 22 \mathrm{H}-1,78$ & $224.26-223.61$ \\
\hline S. heteromorphus LO & $845 \mathrm{~A} 23 \mathrm{H}-4,86 / 23 \mathrm{H}-4,46$ & $237.69-237.29$ \\
\hline R. pseudoumbilicus $\mathrm{FO}$ & $845 \mathrm{~A} 23 \mathrm{H}-6,5 / 23 \mathrm{H}-5,120$ & $239.88-239.53$ \\
\hline
\end{tabular}

Notes: As specified in Table 3.
Table 7. Position of calcareous nannofossil events at Hole 714B.

\begin{tabular}{lll}
\hline \multicolumn{1}{c}{ Event } & \multicolumn{1}{c}{$\begin{array}{c}\text { Core, section } \\
(\mathrm{cm})\end{array}$} & $\begin{array}{c}\text { Depth } \\
(\mathrm{mbsf})\end{array}$ \\
\hline $\begin{array}{l}\text { D. kugleri } \mathrm{LO} \\
\text { D. kugleri } \mathrm{LCO}\end{array}$ & $\begin{array}{l}\text { Not detected } \\
\text { Not detected }\end{array}$ \\
$\begin{array}{l}\text { D. kugleri } \mathrm{FO} \\
\text { C. macintyrei } \mathrm{FO}\end{array}$ & Not reliable & \\
C. nitescens LO & $8 \mathrm{H}-6,40 / 8 \mathrm{H}-5,40$ & $72.5-71.0$ \\
C. premacintyrei $\mathrm{LCO}$ & $9 \mathrm{H}-3,40 / 9 \mathrm{H}-2,40$ & $77.7-76.2$ \\
T. serratus LO & $9 \mathrm{H}-3,40 / 9 \mathrm{H}-2,40$ & $77.7-76.2$ \\
C. floridanus $\mathrm{LCO}$ & $9 \mathrm{H}-4,40 / 9 \mathrm{H}-3,40$ & $79.2-77.2$ \\
T. rugosus FCO & $10 \mathrm{H}-3,40 / 10 \mathrm{H}-2,40$ & $87.3-85.8$ \\
R. pseudoumbilicus $\mathrm{FO}$ & $10 \mathrm{H}-4,40 / 10 \mathrm{H}-3,40$ & $88.8-87.3$ \\
S. heteromorphus $\mathrm{LO}$ & $10 \mathrm{H}-4,40 / 10 \mathrm{H}-3,40$ & $88.8-87.3$ \\
& $10 \mathrm{H}-4,40 / 10 \mathrm{H}-3,40$ & $88.8-87.3$ \\
\hline
\end{tabular}

Notes: As specified in Table 3.

volume). We examined the lower distribution range of $R$. pseudoumbilicus to obtain information about its appearance event. It occurs close to the LO of S. heteromorphus (the boundary between Zones CN4 and CN5) in the equatorial Indian Ocean Site 714 (Fig. 4, and Fornaciari et al., 1990), and at western equatorial Pacific Site 806 (Fig. 5, and Fornaciari et al.,1993). The distribution patterns obtained at Site 806 and at the other Pacific Sites 575 (Fig. 3) and 845 (Fig. 6) show that rare and scattered, but distinctive, large forms of $R$. pseudoumbilicus are present below the extinction level of $S$. heteromorphus. At midlatitude North Atlantic Site 608, Olafsson (1991) reported the FO of $R$. pseudoumbilicus in a different position, as occurring well above the LO of $S$. heteromorphus. If correlated to magnetostratigraphy, the FO of $R$. pseudoumbilicus at Site 608 is associated to the top of Chron 5AAn. This is probably the same event, occurring in the same chronostratigraphic position, as the gradual increase in abundance observed 
Table 8. Position of calcareous nannofossil events at Hole 709C.

\begin{tabular}{|c|c|c|}
\hline Event & $\begin{array}{l}\text { Core, section } \\
(\mathrm{cm})\end{array}$ & $\begin{array}{l}\text { Depth } \\
\text { (mbsf) }\end{array}$ \\
\hline D. quinque ramus LO & $7 \mathrm{H}-6.60 / 7 \mathrm{H}-5.60$ & $62.0-60.5$ \\
\hline A. cimplificus LO & $8 \mathrm{H}-5,60 / 8 \mathrm{H}-4,60$ & $70.2-68.7$ \\
\hline A. amplificus $\mathrm{FO}$ & $10 \mathrm{H}-1.60 / 9 \mathrm{H}-6,60$ & $83.5-81.3$ \\
\hline A. primus $\mathrm{FO}$ & $10 \mathrm{H}-6,60 / 10 \mathrm{H}-5,60$ & $91.0-89.5$ \\
\hline M. convallis LO & $10 \mathrm{H}-6,60 / 10 \mathrm{H}-5.60$ & $91.0-89.5$ \\
\hline D. surculus FCO & $12 \mathrm{H}-1,30 / 11 \mathrm{H}-5,60$ & $102.5-99.1$ \\
\hline D. herggrenii FO & $12 \mathrm{H}-4,30 / 12 \mathrm{H}-3,60$ & $107.0-105.8$ \\
\hline D. pentaradiatus FO & $13 \mathrm{H}-2,60 / 13 \mathrm{H}-1.90$ & $113.9-112.7$ \\
\hline M. convallis FO & $13 \mathrm{H}-3.60 / 13 \mathrm{H}-1.90$ & $113.9-112.7$ \\
\hline D. hamatus LO & $13 \mathrm{H}-2.60 / 13 \mathrm{H}-1.90$ & $113.9-112.7$ \\
\hline D. neohamentus FO & $13 \mathrm{H}-2,90 / 13 \mathrm{H}-2,60$ & $114.2-113.9$ \\
\hline D. prepentaradiatus FO & $13 \mathrm{H}-2,90 / 13 \mathrm{H}-2,60$ & $114.2-113.9$ \\
\hline D. hamatus FO & $1.3 \mathrm{H}-3.60 / 1.3 \mathrm{H}-2.90$ & $115.4-114.2$ \\
\hline C. miopelagicus LO & $13 \mathrm{H}-3.60 / 13 \mathrm{H}-2,90$ & $115.4-114.2$ \\
\hline D. bellius group FO & $1,3 \mathrm{H}-3,60 / 13 \mathrm{H}-2,90$ & $115.4-114.2$ \\
\hline C. calveulus FO & $13 \mathrm{H}-3,60 / 13 \mathrm{H}-2.90$ & $115.4-114.2$ \\
\hline C. coalitus FO & $13 \mathrm{H}-3.90 / 13 \mathrm{H}-3.60$ & $115.7-115.4$ \\
\hline
\end{tabular}

Notes: $\mathrm{FO}=$ first occurrence; $\mathrm{LO}=$ last occurrence; $\mathrm{FCO}=$ first common and continuous occurrence.

Table 9. Position of calcareous nannofossil events at Site 844.

\begin{tabular}{llr}
\hline \multicolumn{1}{c}{ Event } & \multicolumn{1}{c}{$\begin{array}{c}\text { Core, section } \\
(\mathrm{cm})\end{array}$} & $\begin{array}{c}\text { Depth } \\
\text { (mbsf) }\end{array}$ \\
\hline D. quinqueramus LO & $710 \mathrm{~B}-5 \mathrm{H}-6,90 / 5 \mathrm{H}-6,30$ & $43.7-43.1$ \\
A. amplificus LO & $710 \mathrm{~B}-6 \mathrm{H}-2,130 / 6 \mathrm{H}-1,130$ & $47.7-46.2$ \\
A. amplificus FO & $710 \mathrm{~B}-7 \mathrm{H}-1,30 / 6 \mathrm{H}-6,110$ & $54.8-53.5$ \\
A. primus FO & $710 \mathrm{~B}-7 \mathrm{H}-5,30 / 7 \mathrm{H}-4,130$ & $60.8-60.3$ \\
D. surculus FCO & $710 \mathrm{~B}-7 \mathrm{H}-7,15 / 7 \mathrm{H}-6,130$ & $63.65-63.3$ \\
M. convallis LO & $710 \mathrm{~A}-7 \mathrm{H}-4,130 / 7 \mathrm{H}-3,30$ & $63.3-61.8$ \\
D. berggrenii FO & $710 \mathrm{~A}-8 \mathrm{H}-5,130 / 8 \mathrm{H}-5,9$ & $74.4-74.0$ \\
D. bollii LO & $710 \mathrm{~A}-9 \mathrm{H}-1,90 / 9 \mathrm{H}-1,30$ & $77.5-76.9$ \\
D. pentaradiatus FO & $710 \mathrm{~A}-9 \mathrm{H}-2,30 / 9 \mathrm{H}-1,130$ & $78.4-77.9$ \\
M. convallis FO & $710 \mathrm{~A}-9 \mathrm{H}-2,90 / 9 \mathrm{H}-2,30$ & $79.0-78.4$ \\
D. hamatus LO & $710 \mathrm{~A}-9 \mathrm{H}-2,90 / 9 \mathrm{H}-2,30$ & $79.0-78.4$ \\
D. neohamatus FO & $710 \mathrm{~A}-9 \mathrm{H}-4,130 / 9 \mathrm{H}-4,90$ & $82.4-82.0$ \\
D. prepentaradiatus $\mathrm{FO}$ & $710 \mathrm{~A}-9 \mathrm{H}-7,30 / 9 \mathrm{H}-6,130$ & $85.9-85.4$ \\
D. hamatus FO & Not reliable & \\
\hline
\end{tabular}

Notes: As specified in Table 8.

Table 10. Position of calcareous nannofossil events at Site 844 .

\begin{tabular}{|c|c|c|}
\hline Event & $\begin{array}{l}\text { Core, section } \\
(\mathrm{cm})\end{array}$ & $\begin{array}{l}\text { Depth } \\
\text { (mcd) }\end{array}$ \\
\hline D. quinqueramus LO & $844 \mathrm{D} 1 \mathrm{H}-3,150 / 844 \mathrm{C} 4 \mathrm{H}-1,75$ & $33.2-33.0$ \\
\hline A. amplificus LO & $844 \mathrm{C} 4 \mathrm{H}-2,145 / 4 \mathrm{H}-2,125$ & $35.2-35.0$ \\
\hline A. amplificus $\mathrm{FO}$ & $844 \mathrm{~B} 5 \mathrm{H}-2,120 / 5 \mathrm{H}-2,90$ & $39.35-39.05$ \\
\hline A. primus $\mathrm{FO}$ & $844 \mathrm{~B} 5 \mathrm{H}-5,29 / 5 \mathrm{H}-4,120$ & $42.94-42.35$ \\
\hline D. surculus $\mathrm{FO}$ & Not reliable & \\
\hline D. surculus FCO & $844 \mathrm{C} 5 \mathrm{H}-4,25 / 5 \mathrm{H}-3,100$ & $47.6-46.85$ \\
\hline D. berggrenii $\mathrm{FO}$ & $844 \mathrm{~B} 6 \mathrm{H}-5,60 / 6 \mathrm{H}-4,150$ & $53.2-52.6$ \\
\hline D. loeblichii LO & $844 \mathrm{C} 6 \mathrm{H}-1,70 / 844 \mathrm{~B} 6 \mathrm{H} 5,60$ & $54.45-53.2$ \\
\hline D. pentaradiatus $\mathrm{FO}$ & $844 \mathrm{C} 6 \mathrm{H}-3,70 / 6 \mathrm{H}-3,30$ & $57.45-57.05$ \\
\hline D. loeblichii FO & Not reliable & \\
\hline D. bollii LO & $844 \mathrm{C} 6 \mathrm{H}-4,90 / 6 \mathrm{H}-4,50$ & $59.15-58.75$ \\
\hline D. prepentaradiatus LO & Not reliable & \\
\hline D. hamatus LO & $844 \mathrm{C} 6 \mathrm{H}-5,30 / 844 \mathrm{~B} 7 \mathrm{H}-1,120$ & $60.05-59.9$ \\
\hline D. neohamatus FO & $844 \mathrm{~B} 7 \mathrm{H}-4,27 / 7 \mathrm{H}-3,121$ & $63.47-62.91$ \\
\hline D. hamatus FO & $844 \mathrm{C} 7 \mathrm{H}-5,50 / 7 \mathrm{H}-5,25$ & $70.75-70.5$ \\
\hline D. exilis LCO & $844 \mathrm{C} 7 \mathrm{H}-5,75 / 7 \mathrm{H}-5,50$ & $71.0-70.75$ \\
\hline C. miopelagicus LO & $844 \mathrm{C}-7 \mathrm{H}-6,2 / 7 \mathrm{H}-5,125$ & $71.77-71.5$ \\
\hline C. calyculus FO & $844 \mathrm{C} 7 \mathrm{H}-6,100 / 7 \mathrm{H}-6,25$ & $72.75-72.0$ \\
\hline D. bellus group $\mathrm{FO}$ & $844 \mathrm{C} 7 \mathrm{H}-7,25 / 7 \mathrm{H}-6,100$ & $73.5-72.75$ \\
\hline D. calcaris FO & $844 \mathrm{C} 7 \mathrm{H}-7,70 / 7 \mathrm{H}-7,25$ & $73.95-73.5$ \\
\hline
\end{tabular}

Notes: As specified in Table 3.

at Pacific Site 845 (Fig. 6). The data here reported confirm the ecological control on the distribution of $R$. pseudoumbilicus and the regional value of its $\mathrm{FO}$ as a biostratigraphic marker in tropical/equatorial areas, as previously suggested by Fornaciari et al. (1993).

As commented below, the temporary disappearance of these large reticulofenestrids was observed both in the tropical Indian and Pacific
Table 11. Position of calcareous nannofossil events at Site 848.

\begin{tabular}{|c|c|c|}
\hline Event & $\begin{array}{l}\text { Core, section } \\
(\mathrm{cm})\end{array}$ & $\begin{array}{l}\text { Depth } \\
\text { (mcd) }\end{array}$ \\
\hline D. quinqueramus LO & $848 \mathrm{D} 6 \mathrm{H}-6,50 / 848 \mathrm{C} 6 \mathrm{H}-7,8$ & $59.4-58.97$ \\
\hline A. amplificus LO & $848 \mathrm{C} 7 \mathrm{H}-2,50 / 7 \mathrm{H}-2,25$ & $62.79-62.54$ \\
\hline A. amplificus FO & $848 \mathrm{C} 7 \mathrm{H}-7,50 / 7 \mathrm{H}-7,20$ & 70.29 \\
\hline A. primus $\mathrm{FO}$ & $848 \mathrm{C} 8 \mathrm{H}-6,140 / 8 \mathrm{H}-6,80$ & $80.54-79.94$ \\
\hline D. surculus FCO & $848 \mathrm{C} 8 \mathrm{H}-6,140 / 8 \mathrm{H}-6,80$ & $80.54-79.94$ \\
\hline D. surculus FO & Not reliable & \\
\hline D. berggrenii $\mathrm{FO}$ & $848 \mathrm{~B} 9 \mathrm{H}-3,119 / 9 \mathrm{H}-3,100$ & $82.49-82.3$ \\
\hline D. pentaradiatus $\mathrm{FO}$ & Not reliable & \\
\hline M. convallis LO & Not reliable & \\
\hline M. convallis $\mathrm{FO}$ & $848 \mathrm{~B} 10 \mathrm{H}-2, / 10 \mathrm{H}-2,120$ & $91.8-91.5$ \\
\hline D. hamatus LO & $848 \mathrm{C} 10 \mathrm{H}-1,125 / 848 \mathrm{~B} \mathrm{IOH}-4,10$ & $93.57-93.4$ \\
\hline D. neohamatus $\mathrm{FO}$ & $848 \mathrm{C} 10 \mathrm{H}-2,145 / 10 \mathrm{H}-2,100$ & $95.27-94.82$ \\
\hline D. ham & $848 \mathrm{C} 10 \mathrm{H}-5,50 / 10 \mathrm{H}-5,30$ & $98.82-98.62$ \\
\hline C. miopelagicus $\mathrm{LO}$ & $848 \mathrm{C} 10 \mathrm{H}-5,75 / 10 \mathrm{H}-5,50$ & $99.07-98.82$ \\
\hline D. bellus group FO & $848 \mathrm{C} 10 \mathrm{H}-6,100 / 848 \mathrm{~B} 11 \mathrm{H}-1,50$ & $100.82-100.59$ \\
\hline D. exilis LCO & Not reliable & \\
\hline D. calcaris $\mathrm{FO}$ & $848 \mathrm{~B}-11 \mathrm{H}-3,90 / 11 \mathrm{H}-3,40$ & $103.99-103.49$ \\
\hline
\end{tabular}

Notes: As specified in Table 3.

Table 12. Position of calcareous nannofossil events at Hole 711B.

\begin{tabular}{llc}
\hline \multicolumn{1}{c}{ Event } & \multicolumn{1}{c}{$\begin{array}{c}\text { Core, section } \\
(\mathrm{cm})\end{array}$} & \multicolumn{1}{c}{$\begin{array}{c}\text { Depth } \\
(\mathrm{mbsf})\end{array}$} \\
\hline D. quinqueramus $\mathrm{LO}$ & $4 \mathrm{H}-1,25 / 3 \mathrm{H} \mathrm{CC}$ & $21.65-21.4$ \\
A. amplificus $\mathrm{LO}$ & $4 \mathrm{H}-1,100 / 4 \mathrm{H}-1,75$ & $22.4-22.15$ \\
A. amplificus $\mathrm{FO}$ & $4 \mathrm{H}-5,25 / 4 \mathrm{H}-4,150$ & $27.65-27.4$ \\
A. primus $\mathrm{FO}$ & Not reliable & \\
D. surculus FCO & $5 \mathrm{H}-3,50 / 5 \mathrm{H}-2,125$ & $34.5-33.75$ \\
D. berggrenii FO & $5 \mathrm{H}-4,145 / 5 \mathrm{H}-4,125$ & $36.95-36.75$ \\
\hline
\end{tabular}

Notes: As specified in Table 8.

Table 13. Position of calcareous nannofossil events at Site 853 .

\begin{tabular}{|c|c|c|}
\hline Event & $\begin{array}{l}\text { Core, section } \\
(\mathrm{cm})\end{array}$ & $\begin{array}{l}\text { Depth } \\
\text { (med) }\end{array}$ \\
\hline$D$ guinqueramus $\mathrm{LO}$ & $853 \mathrm{D}-4 \mathrm{H}-1,140 / 4 \mathrm{H}-1,100$ & $33.35-33.95$ \\
\hline A. amplificus LO & $853 \mathrm{~B}-5 \mathrm{H}-5,140 / 5 \mathrm{H}-5,65$ & $37.9-37.1$ \\
\hline A amplificus FO & $853 \mathrm{~B}-6 \mathrm{H}-4,27 / 6 \mathrm{H}-4,65$ & $46.1-45.7$ \\
\hline$R$. pseudoumbilicus $\mathrm{FO}$ & $853 \mathrm{~B}-6 \mathrm{H}-6,65 / 6 \mathrm{H}-5,65$ & $49.1-47.6$ \\
\hline A. primus FO & $853 \mathrm{D}-6 \mathrm{H}-2,100 / 6 \mathrm{H}-2,140$ & $55.8-55.4$ \\
\hline$D$ surculus $\mathrm{FCO}$ & $853 \mathrm{D}-6 \mathrm{H}-7,90 / 6 \mathrm{H}-7,70$ & $62.7-61.1$ \\
\hline$D$ berggrenii $\mathrm{FO}$ & $853 \mathrm{D}-7 \mathrm{H}-4,120 / 7 \mathrm{H}-4,140$ & $69.4-69.6$ \\
\hline D neohamatus FO & $853 \mathrm{D}-7 \mathrm{H}-7,30 / 7 \mathrm{H}-6,140$ & $73.0-72.6$ \\
\hline
\end{tabular}

Notes: As specified in Table 8.

oceans within a long stratigraphic interval in the late Miocene, corresponding to the interval between the LO of Discoaster hamatus and the FO of Amaurolithus amplificus.

\section{LCO of Cyclicargolithus floridanus (3)}

The LO of Cyclicargolithus floridanus has been proposed by Bukry (1973) as an alternative event to the FO of Discoaster kugleri for recognizing the $\mathrm{CN} 5 \mathrm{a} / \mathrm{CN} 5 \mathrm{~b}$ zonal boundary. Its final distribution pattern was established in detail at various sites (Olafsson, 1989, 1991; Fornaciari et al., 1990, 1993).

We studied the final range of $C$. floridanus at Sites 575 (Fig. 3), 714 (Fig. 4), 806 (Fig. 5), and 845 (Fig. 6). Our data, as well as the previous studies, indicate that the distribution pattern of $C$. floridanus in its final range is different at each site. Specifically, in low-latitude areas (Sites 574, 575, 714, 806, 845), we recorded what appears to be the LO of the species shortly above the LO of S. heteromorphus. At mid-latitude North Atlantic Site 608, Olafsson (1991) observed the exit of $C$. floridanus at a much higher stratigraphic level (Fig. 17), associated with the lower part of Chron 5r. However, Olafsson 

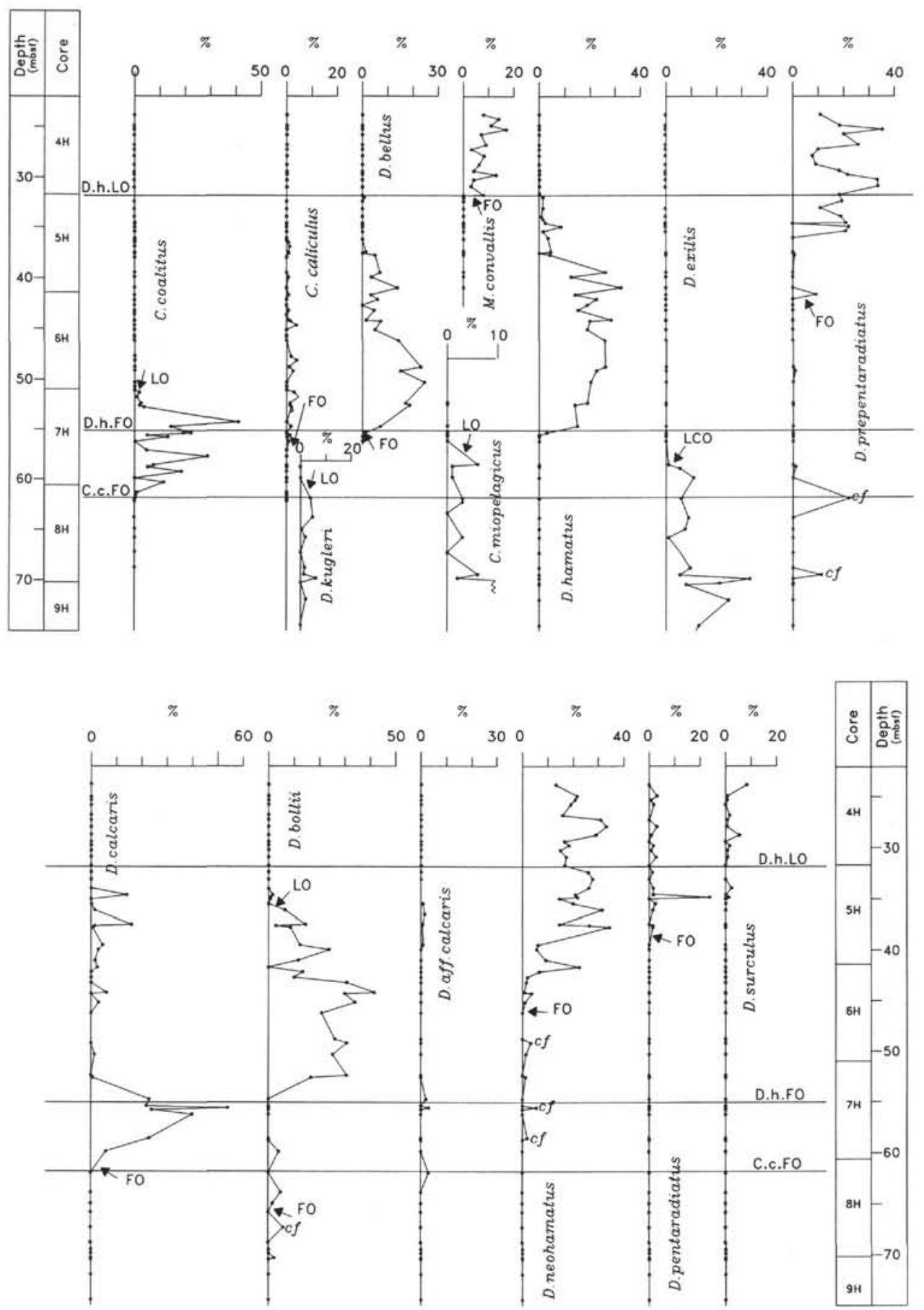

Figure 8. Abundance patterns of middle and late Miocene selected calcareous nannofossils at Hole 714A. (mbsf) $=$ meters below seafloor. Notation as specified in Figure 3. D.h. $=$ Discoaster hamatus $;$ C.c. $=$ Catinaster coalitus .

showed a marked decrease in abundance occurring within Chron $5 \mathrm{AAr}$, the same time interval during which the species became excluded from low-latitude areas (Figs. 3, 4, 5, and 7). It is interesting to note that in the high-resolution sampling at Site 845 (Fig. 6), we detected short intervals of $C$. floridanus up to the topmost part of Chron 5An (i.e., at a level close to the final exit of the species at Site 608 in a mid-latitude area. On the basis of these data, it may be useful to distinguish a LCO (last common and continuous occurrence) event for the apparent extinction of $C$. floridanus at low latitudes and an LO event for the probably true extinction of the species.
We conclude, in agreement with Fornaciari et al. (1993), that the stratigraphic position of the $C$. floridanus disappearance varies latitudinally and should be used with caution for long distance correlation.

\section{LO of Discoaster signus (4)}

The easily recognized Discoaster signus characterizes the nannofossil assemblage in the lower part of the middle Miocene sequence, appearing close to the LO of Helicosphaera ampliaperta $(\mathrm{CN} 3 / \mathrm{CN} 4$ and NN4/NN5 boundaries) in both the tropical Indian Ocean (Rio et 


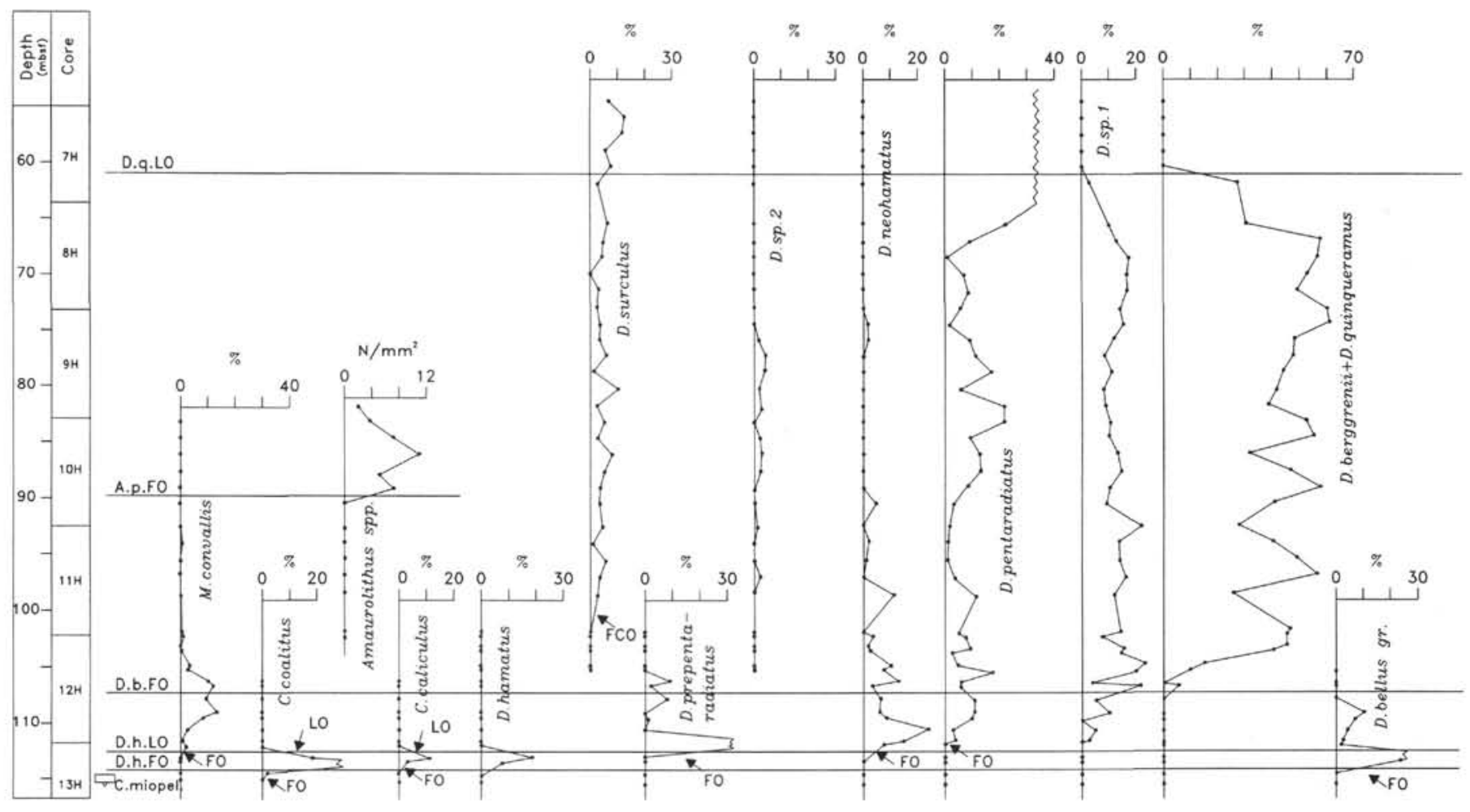

Figure 9. Abundance patterns of middle and late Miocene selected calcareous nannofossils at Hole 709C. (mbsf) $=$ meters below seafloor. Notation as specified in Figure 3. D.q. = Discoaster quinqueramus; A.p. = Amaurolithus primus; D.b. $=$ Discoaster berggrenii $;$ D.h. $=$ Discoaster hamatus; C.c. $=$ Catinaster coalitus .

al., 1990a) and eastern equatorial Pacific Ocean (Raffi and Flores, this volume). As no detailed data are available on its extinction (located within Zone NN6 by Perch-Nielsen, 1985), we have established the final range of the species at Site 845 (Fig. 6). In this sequence, $D$. signus is well represented and becomes extinct during CN5a (NN6) time, as previously indicated by Bukry (1973) and Perch-Nielsen (1985), and observed at Site 844 (Raffi and Flores, this volume).

\section{Triquetrorhabdulus rugosus FCO (5) and Triquetrorhabdulus rugosus-Triquetrorhabdulus rioensis/Triquetrorhabdulus serratus Reversal in Abundance}

Bukry (1973) suggested that the FO of Triquetrorhabdulus rugosus was an additional marker for distinguishing the top of Zone CN4. Fornaciari et al. (1990) in the tropical Indian Ocean and Olafsson (1991) at mid-latitude North Atlantic Site 608 showed that the FO of T. rugosus is a biostratigraphically useful event. They defined this FO as a sharp rise in abundance at the beginning of the T. rugosus range. Olafsson (1989) reported the quantitative distribution patterns of $T$. rugosus (together with $T$. rioensis) and of Triquetrorhabdulus serratus at Site 574 in the equatorial Pacific Ocean and Hole 667A in the Atlantic Ocean. At both sites, a reversal in abundance between $T$. rugosus $-T$. rioensis and $T$. serratus was evidenced. Namely, $T$. rugosus $-T$. rioensis increased in abundance at the extinction level of $T$. serratus.

Here, we report the distribution patterns of these species at Indian Ocean Site 714 (Fig. 4) and at equatorial Pacific Site 575 (Fig. 3), 806 (Fig. 5), and 845 (Fig. 6). At these sites we recorded the same relative abundance variations between $T$. serratus and $T$. rugosus-T. rioensis as those observed by Olafsson (1989). Moreover, in the considered sections, the first occurrence of $T$. rugosus was recorded in the same position with respect to the adjoining biohorizons (Fig. 17). Note, however, the short spacing between the LO of S.heteromorphus and the FO of $T$. rugosus at Site 714 (Figs. 4 and 17). Most probably a hiatus is present at this site, which was not inferred by Rio et al., (1990a).
As regards the magnetostratigraphic position of the FO of $T$. rugosus, Berggren et al. (1985) associated the event to Chron 5AC, referring to data of Miller et al. (1985) at Atlantic Sites 558 and 563. As the interpretation of magnetostratigraphy of Site 558 is difficult (see Fig. 1 in Miller et al., 1985), we do not consider valid this succession for any calibration in the time interval of interest. In the new interpretation of the magnetostratigraphy of Site 563 of Miller et al. (in press), the FO of T. rugosus is associated to Chron 5AAn, as it is at Site 608, according to the data of Gartner (1992). When compared at the different sites considered, the magnetostratigraphic position of the FO of T. rugosus is different. At Site 845 (Fig. 6), the beginning of continuous distribution of $T$. rugosus is associated to Chron $5 \mathrm{Ar}$ (Subchron 5Ar.1r).

Most probably this diachroneity of about 0.20 .4 m.y. depends on different event definitions used by the different authors. In fact, although we found rare specimens of $T$. rugosus just above Chron 5AAn (see Fig. 6), we consider the rise in abundance and the beginning of continuous occurrence (FCO) a more distinct event and a reproducible datum. From the available data, it appears that $T$. rugosus FCO can be a good biochronostratigraphic correlation tool for long distance correlations.

\section{LCO of Calcidiscus premacintyrei (6)}

Theodoridis (1984) and Gartner (1992) suggested the LO of the distinctive Calcidiscus premacintyrei as a potentially useful marker for subdividing the long interval between the LO of $S$. heteromorphus and the FO of Catinaster coalitus. We have established the distribution pattern of $C$. premacintyre $i$ in its upper range, at Sites 575 (Fig. 3), 806 (Fig. 5), 845 (Fig. 6), and 714 (Fig. 7). In all the investigated sections, the LCO of the species seems to occur in the same biostratigraphic position, slightly below the LO of $C$. nitescens. Few specimens, discontinuously occurring, are present above this LCO at Sites 806, 714, and 845 (Figs. 5, 6, and 7). We consider these discontinuous occurrences as the genuine tail of distribution of the species, not related to rework- 

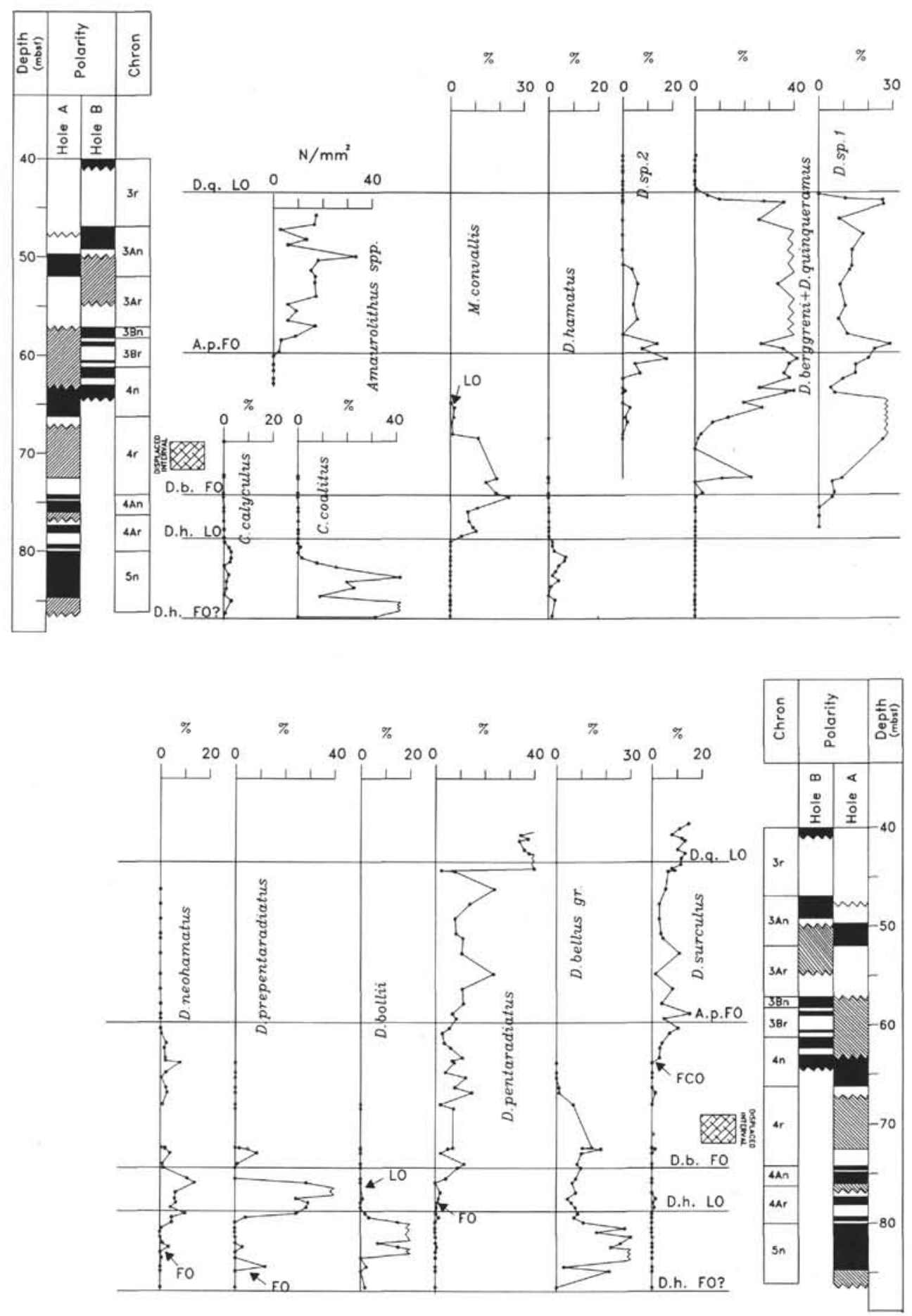

Figure 10. Abundance patterns of middle and late Miocene selected calcareous nannofossils at Site $710 .(\mathrm{mbsf})=$ meters below seafloor. Notation as specified in Figure 3. D.q. = Discoaster quinqueramus; A.p. = Amaurolithus primus; D.b. $=$ Discoaster berggrenii $;$ D.h. $=$ Discoaster hamatus .

ing. As shown in Fig. 6, at Site 845, the LCO of $C$. premacintyrei is associated with the middle part of Subchron C5An.2n. At Site 608, Gartner (1992) correlated the LO of the species with the upper part of Chron 5 An (Subchron C5An.1n) (Fig. 17). Most probably, the event of Gartner refers to the absolute LO of the species, which in fact is associated with the upper part of Chron 5An at Site 845 as well (Fig. 6). As indicated by the obtained results, there is a good chance that the final exit of $C$. premacintyrei can be a useful event for long distance correlations, when more data will be available in wider geographic areas.

\section{LOs of Triquetrorhabdulus serratus (7) and Coronocyclus nitescens (8)}

In the tropical Indian Ocean (Fornaciari et al., 1990) and western equatorial Pacific Ocean (Fornaciari et al., 1993), the LO of Coronocyclus nitescens is considered a good biostratigraphic event, useful for subdividing the interval between the LO of $S$. heteromorphus and the FO of C. coalitus (CN5 of Bukry, 1973) and correlating lowlatitude oceanic sediments. 


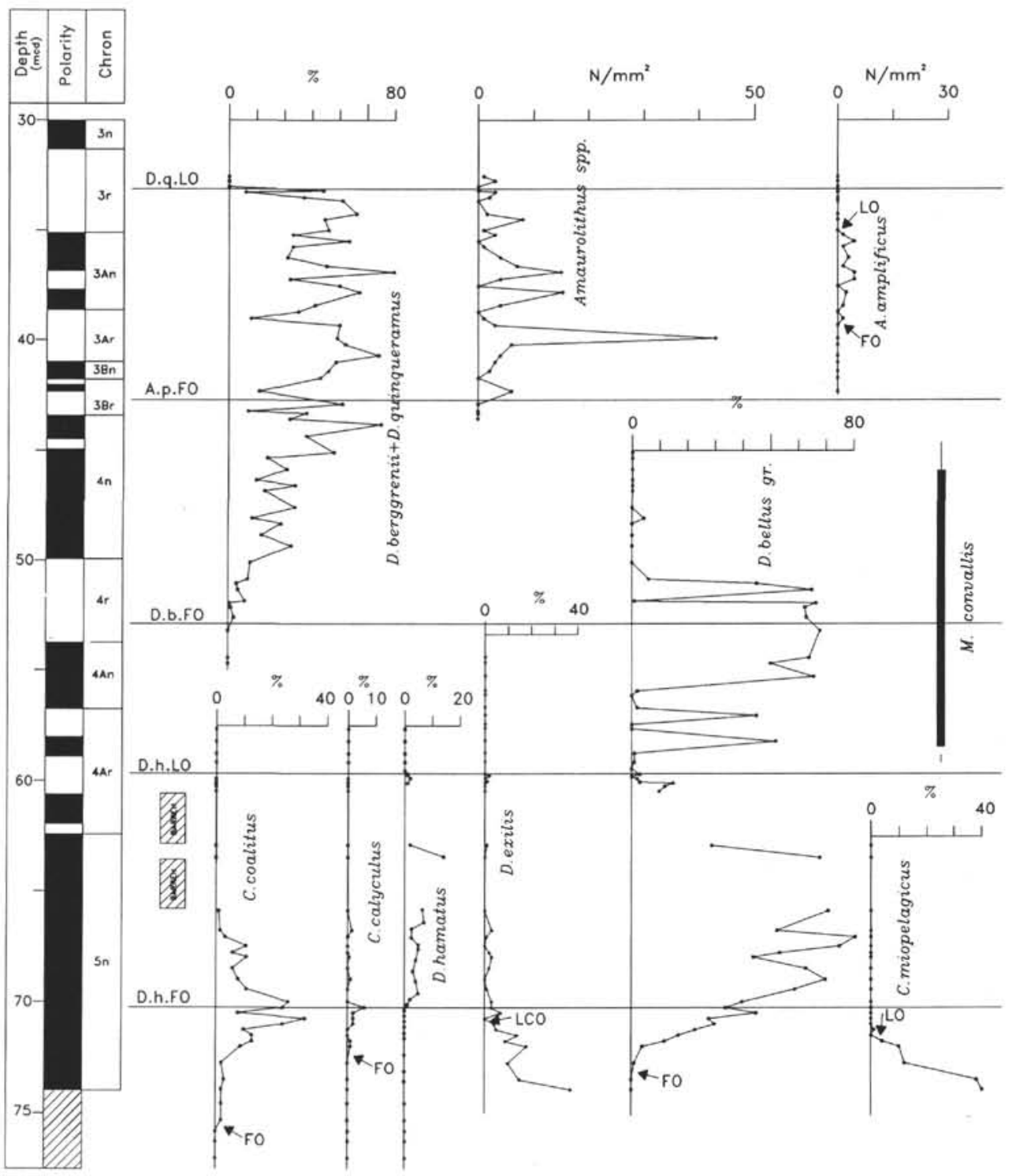

Figure 11. Abundance patterns of middle and late Miocene selected calcareous nannofossils at Site $844 .(\mathrm{mcd})=$ meters composite depth. Notation as specified in Figure 3. D.q. = Discoaster quinqueramus; A.p. = Amaurolithus primus; D.b. $=$ Discoaster berggrenii; D.h. = Discoaster hamatus. Magnetostratigraphy from site chapters in Mayer, Pisias, Janecek, et al. (1992) and Schneider (this volume).

New data obtained at Pacific Sites 575 (Fig. 3) and 845 (Fig. 6) confirm the reliability of this event. The last specimens of $C$. nitescens were easily detected and occurred together with the last representatives of $T$. serratus. These two extinction events together provide a clear biostratigraphic signal, which we observed even at Sites 806 (Fig. 4) and 714 (Figs. 4 and 7). At the eastern equatorial Pacific Site 845 , the LOs of $T$. serratus and $C$. nitescens were correlated to the magnetostratigraphic record. The two events occur in the middle part of Chron 5An, namely in the upper part of Subchron 5An.2n. Even at mid-latitude Site 806, the distribution range of $C$. nitescens seems to end within Chron 5An (see Fig. 7 in Olafsson, 1991), although at this site, the distribution pattern of the species is not distinct, as it shows low abundance and scattered occurrence in the upper part of the range.

\section{FO of Calcidiscus macintyrei (9)}

The FO of Calcidiscus macintyrei was proposed by Bukry (1973) as alternative event for recognizing the $\mathrm{CN} 3 / \mathrm{CN} 4$ boundary. How- ever, highly contradictory biostratigraphic positions of this event are reported in the literature (see Rio et al., 1990a, and Olafsson, 1991). Certainly, these contradictions are partly related to different taxonomic concepts used in detecting of the nominate species. In this study, we ascribe to $C$. macintyrei specimens equal to or larger than $11 \mu \mathrm{m}$, namely those morphotypes whose extinction is a well-known early Pleistocene biostratigraphic event (Backman and Shackleton, 1983; Fornaciari et al., 1990; Raffi et al., in press).

Data from tropical Indian Ocean (Site 714) and equatorial Pacific Ocean (Sites 806,575 , and 845 ) indicate contradictory results about the position of the FO of Calcidiscus $\geq 11 \mu \mathrm{m}$. At Site 714 (Fig. 7), as well as at other Leg 115 sites (Rio et al., 1990), this Calcidiscus consistently occurs in upper CN5 Zone, just above the LO of C. nitescens.

At the Pacific sites considered, the position of the event is variable and its recognition is difficult in some sequences. At Site 845, $C$. macintyrei $(\geq 11 \mu \mathrm{m})$ was found in spotted samples in the interval close to the LO of $C$. nitescens (Raffi and Flores, this volume). It was very rare and sporadic above (Fig. 16), where it was expected to be as 


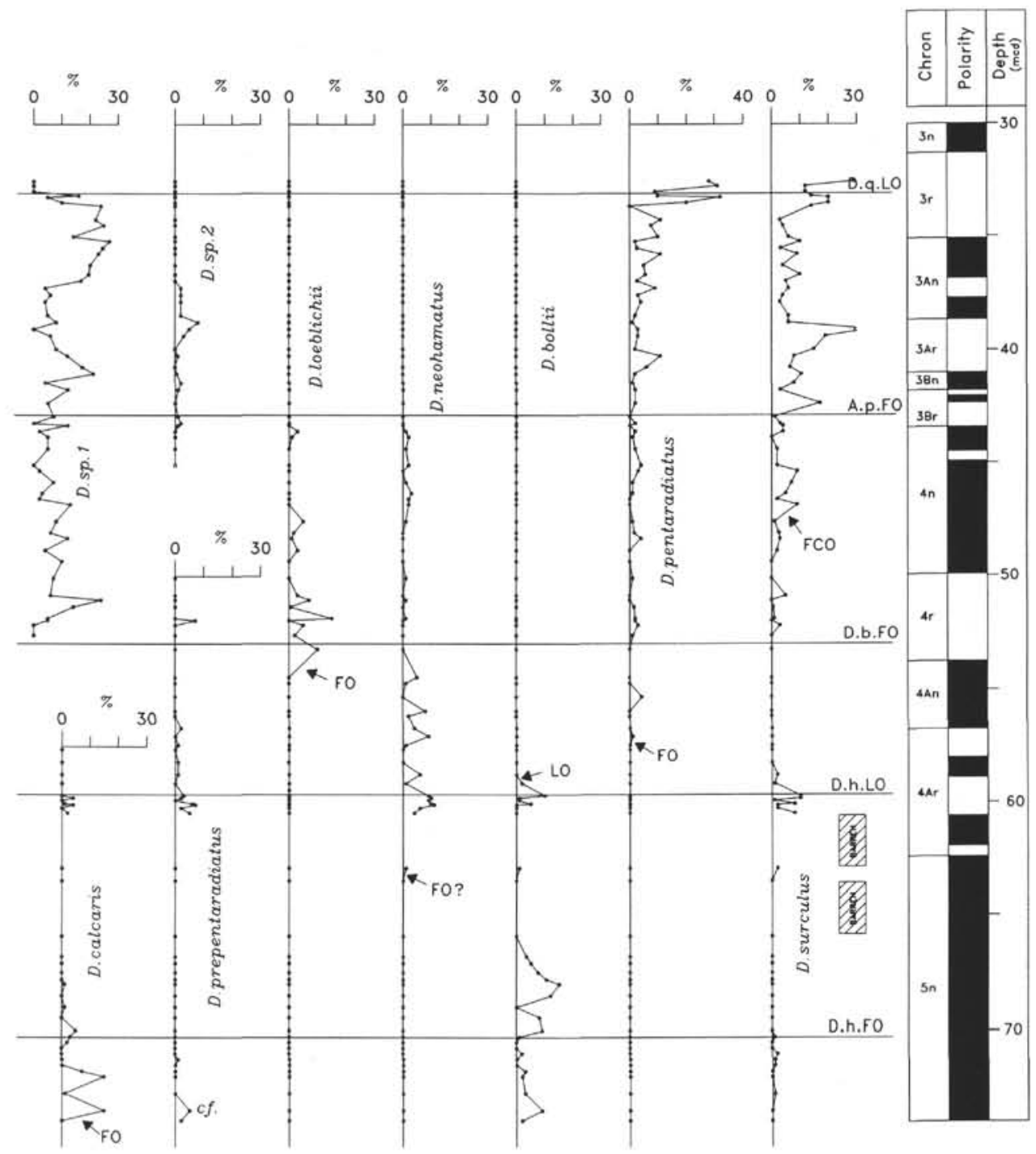

Figure 11 (continued).

common as at Site 714, so that we could not report in Figure 5 any significative distribution pattern of $C$. macintyrei . A biometric analysis of Calcidiscus size was conducted in the lower part of the scattered range of $C$. macintyrei and is shown in Figure 16. The result confirms what was previously suggested by Fornaciari et al. (1990) about the absence of bimodal size distribution in the Calcidiscus population during the middle Miocene.

Low abundance and sporadic occurrence of $C$. macintyrei in the lowermost range was observed even at Site 806 (Fornaciari et al., 1993, and unpubl. data), whereas at Site 575 (Fig. 2) this Calcidiscus seems to appear below the $C$. nitescens LO and consistently occurs upward, although with low abundance.

The obtained results indicate that the biostratigraphic position of the FO of $C$. macintyrei $(\geq 11 \mu \mathrm{m})$ probably is controlled by biogeographic factors, not only by taxonomic ambiguities, which make this event a poor marker as regards reliability and correlation potentiality.

The FO, FCO, and LCO of Discoaster kugleri (events 10,11, and 13).

We monitored the range of Discoaster kugleri with an attempt to clarify the biostratigraphic value of the species, which is a wellknown "controversial" marker in the middle Miocene interval. The FO of the species, which defines the boundary CN5a/CN5b (Bukry, 1973), is considered a weak marker by many authors (e.g., Gartner and Chow, 1985; Fornaciari et al., 1990). Gartner (1992) stated that the range of $D$. kugleri remains uncertain because the species is gen- erally very rare in oceanic areas. Studying the mid-latitude Site 608 , he observed a short range of D. kugleri within Chron $5 \mathrm{r}$ and considered those FOs and LOs as useful events.

The species, indeed, is a "sporadic, tropical species" (Bukry, 1973, p. 692), but it seemed the only criterion for dividing Zone $\mathrm{CN} 5$, which spans a time interval of about 3.0 m.y. (Fig. 17). We report the distribution range of D. kugleri at tropical Indian Ocean Site 714 (Fig. 7) and equatorial Pacific Site 845 (Fig. 6). This last sequence turned out to be the best available sequence for evaluating the actual range of D. kugleri. At this site, the species showed a long interval of total distribution. If correlated to magnetostratigraphy, it extends from the upper part of Subchron $5 \mathrm{An} .2 \mathrm{n}$ to the lower part of Subchron $5 \mathrm{n} .2 \mathrm{n}$ (namely an interval of more than $1 \mathrm{~m} . \mathrm{y}$. using the chronology of Cande and Kent, 1992). These data confirm the weakness of $D$. kugleri FO datum. D. kugleri appears with very rare specimens (less than $2 \%$ of the total discoasterid assemblage) and is present only in scattered samples along the lower part of its range. The lowermost observed specimens occurred concomitantly with the LOs of $C$. nitescens and C. premacintyrei. D. kugleri became common and continuously present only in a short interval, which was detected and correlatable in the equatorial Pacific (Raffi and Flores, this volume). This interval of common and continuous presence of D. kugleri is associated with the lower part of Chron $5 \mathrm{r}$, and terminates in correspondence with Subchron $5 \mathrm{r} .2 \mathrm{n}$. This is the same magnetostratigraphic 


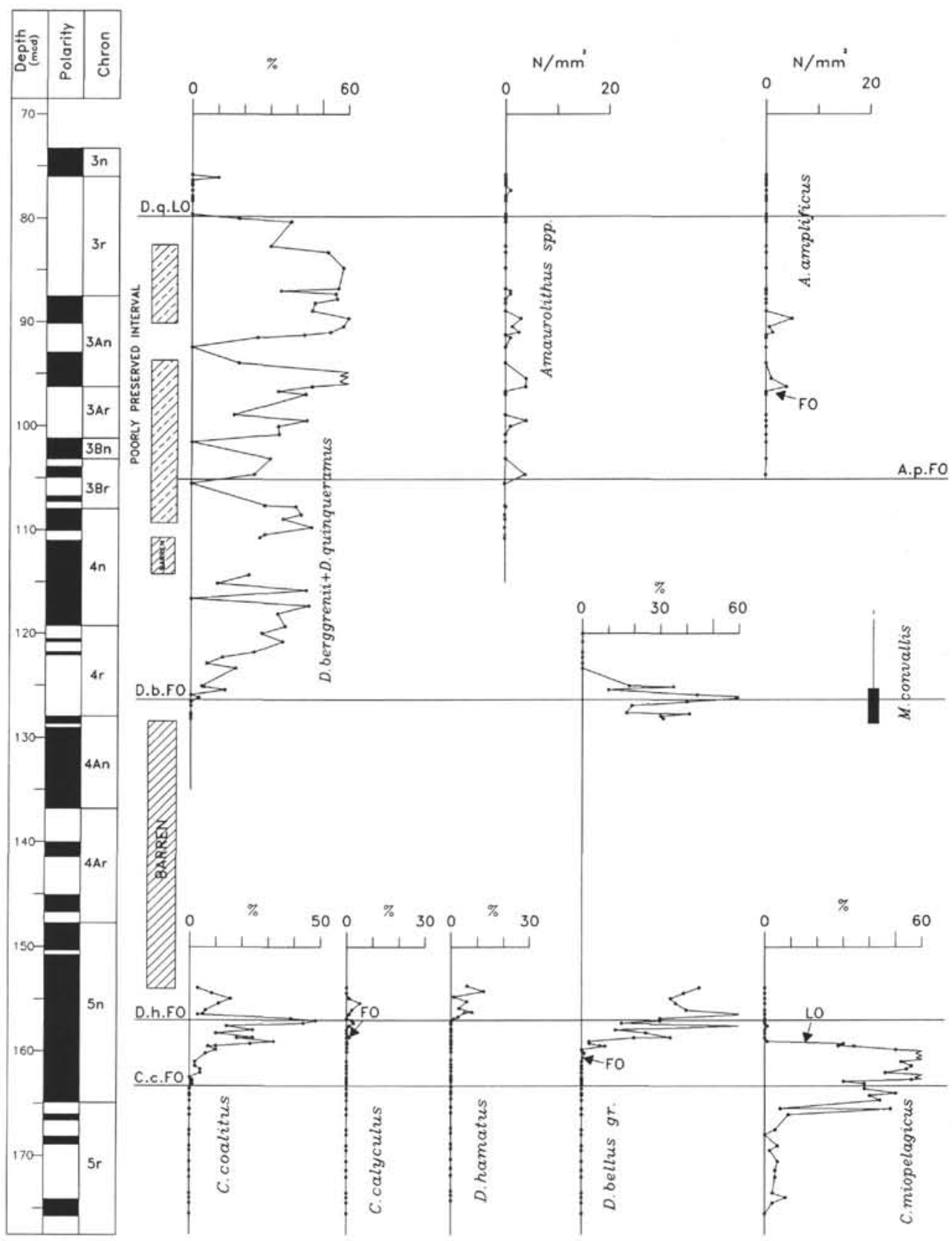

Figure 12. Abundance patterns of middle and late Miocene selected calcareous nannofossils at Site 845. (mcd) $=$ meters composite depth. Notation as specified in Figure 3. D.q. = Discoaster quinqueramus; A.p. = Amaurolithus primus; D.b. = Discoaster berggrenii $;$ D.h. = Discoaster hamatus; C.c. $=$ Catinaster coalitus. Magnetostratigraphy from site chapters in Mayer, Pisias, Janecek, et al. (1992) and Schneider (this volume).

position of $D$. kugleri short range observed at mid-latitude North Atlantic Site 608 (Gartner, 1992).

At tropical Indian Ocean Site 714, D. kugleri FO was detected with many uncertainties (Figs. 7 and 8) owing to poor preservation of discoasterids in that interval (see Fornaciari et al., 1990). However, a short range with common $D$. kugleri was evidenced even in this sequence, probably correlatable with what was observed in the Pacific and midlatitude North Atlantic.

Further investigations regarding the range of $D$. kugleri in the different oceanic areas will be needed to test the usefulness of its FCO and LCO as biostratigraphic markers for further subdividing Zone $\mathrm{CN} 5$. Anyhow, we consider these two events potentially useful correlation lines between geographic distances.

\section{FO of Discoaster bollii (12)}

The appeaerance of Discoaster bollii was considered by Bukry (1973) in his discussion concerning markers for defining Subzones $\mathrm{CN} 5 \mathrm{a}$ and $\mathrm{CN} 5 \mathrm{~b}$. He indicated that this event occurs above the FO of D. kugleri, namely within Subzone CN5b, and therefore considered it unsuitable as an auxiliary marker for defining the base of $\mathrm{CN} 5 \mathrm{~b}$, as suggested by Ellis (1981).

In the eastern equatorial Pacific (Leg 138 sequences), the range of D. bollii was not completely documented owing to the presence of dissolution intervals barren of nannofossils at Sites 844 and 845 (Figs. 11 and 12) and to bad preservation (strong overgrowth) of discoasterids at Site 848 (Fig. 13). We determined the basal range of 


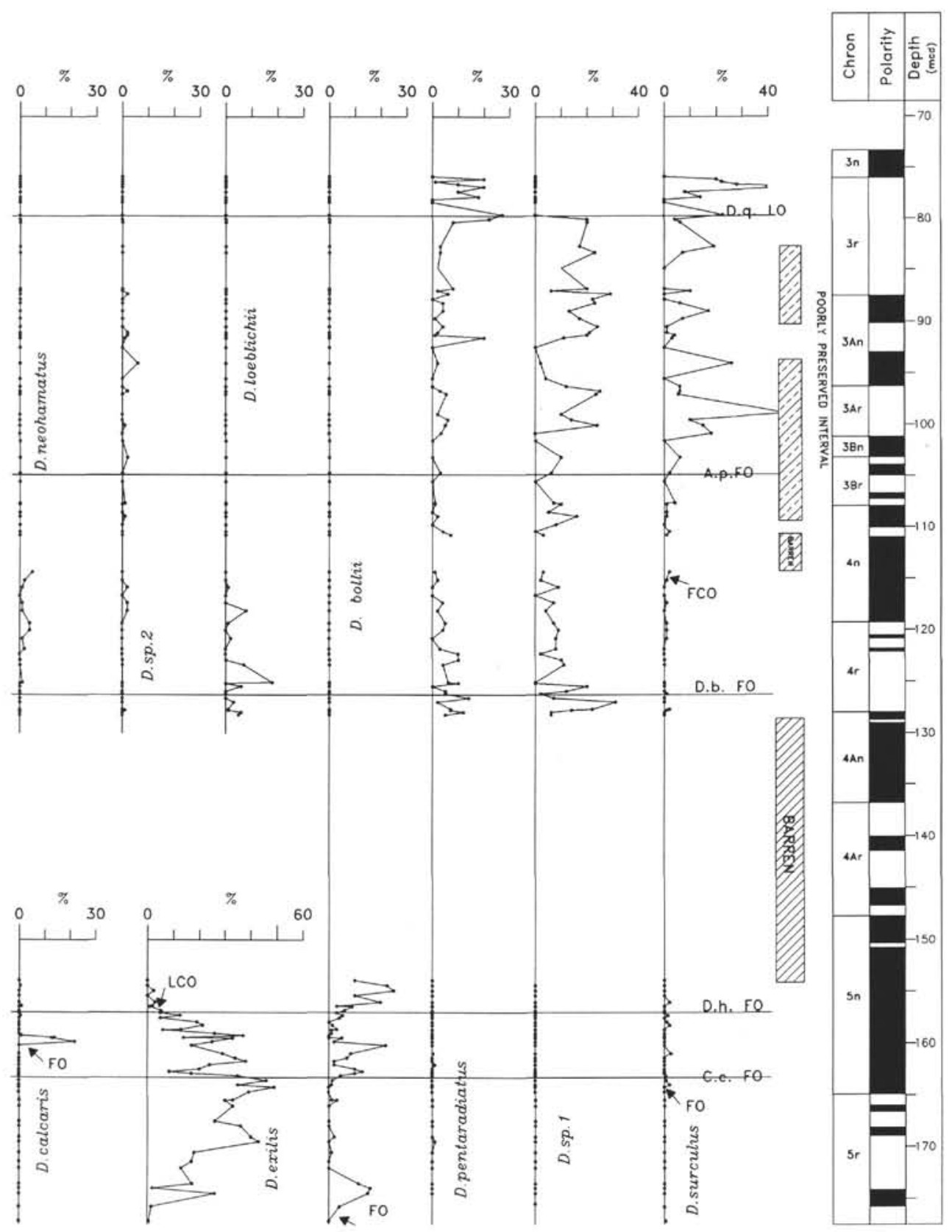

Figure 12 (continued).

D. bollii at Site 845 (Fig. 12) and at equatorial Indian Ocean Site 714 (Fig. 8). At both sites, the stratigraphic position of D. bollii FO conforms with Bukry's statement. At Site 845, the event can be correlated to Chron $5 \mathrm{r}$ (at the base of Subchron $5 \mathrm{r} .2 \mathrm{n}$ ). No data for the FO of $D$. bollii are available from mid-latitude Site 608 .

\section{LO of Discoaster kugleri (14)}

Bukry (1973) suggested as the secondary criterion of definition of the boundary CN5b/CN6 (Discoaster kugleri Subzone/Catinaster coalitus Zone) the LO of the nominate species.

The distribution pattern of D. kugleri at Site 845 (Fig. 6) shows that the highest rare specimens of $D$. kugleri occur together with the lowest specimens of $C$. coalitus. A similar LO event of D. kugleri was observed at Site 115-714 (Fig. 8), thus confirming the indication of Bukry (1973).

However, in both Indian and Pacific sequences, the species is very rare and discontinuous or absent in some intervals of its upper range, as it is in its lower range. The true LO generally is detected with difficulty (Raffi and Flores, this volume); therefore, this event does not represent a useful auxiliary marker for defining the base of CN6, nor is it a reliable correlation line.

\section{FO of Catinaster coalitus (15)}

The FO of Catinaster coalitus is the primary criterion suggested by Bukry (1973) and Martini (1971) for defining the base of Zone CN6 (NN8). This usage has been proved reliable for low-latitude assemblages (Bukry, 1973), because the species is scattered to absent at mid-high latitude sites (i.e., at Site 608, Olafsson, 1991) and in the Mediterranean region (Theodoridis, 1984).

The distribution patterns of $C$. coalitus (Figs. 6, 8, 10, and 12) indicate that the event is easily detected at low-latitude sequences and maintains a consistent biostratigraphic position among the studied sequences (Fig. 17). However, even at low-latitudes, the FO of $C$. coalitus is sometimes undetectable, as shown in some Leg $138 \mathrm{se}$ - 

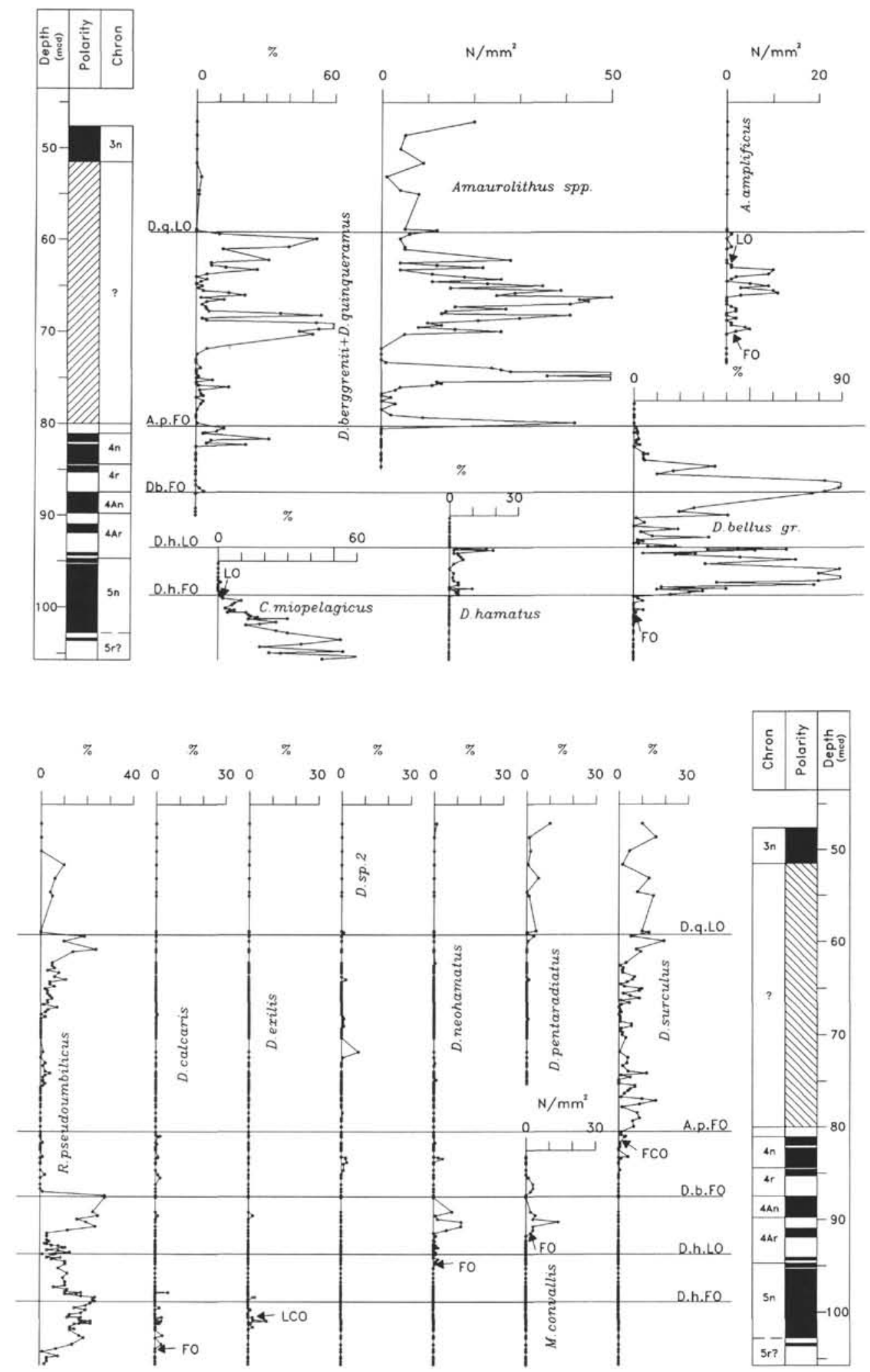

Figure 13. Abundance patterns of middle and late Miocene selected calcareous nannofossils at Site 848. (mcd) = meters composite depth. Notation as specified in Figure 3. D.q. = Discoaster quinqueramus; A.p. = Amaurolithus primus; D.b. = Discoaster berggrenii D.h. = Discoaster hamatus. Magnetostratigraphy from site chapters in Mayer, Pisias, Janecek, et al. (1992) and Schneider (this volume). 


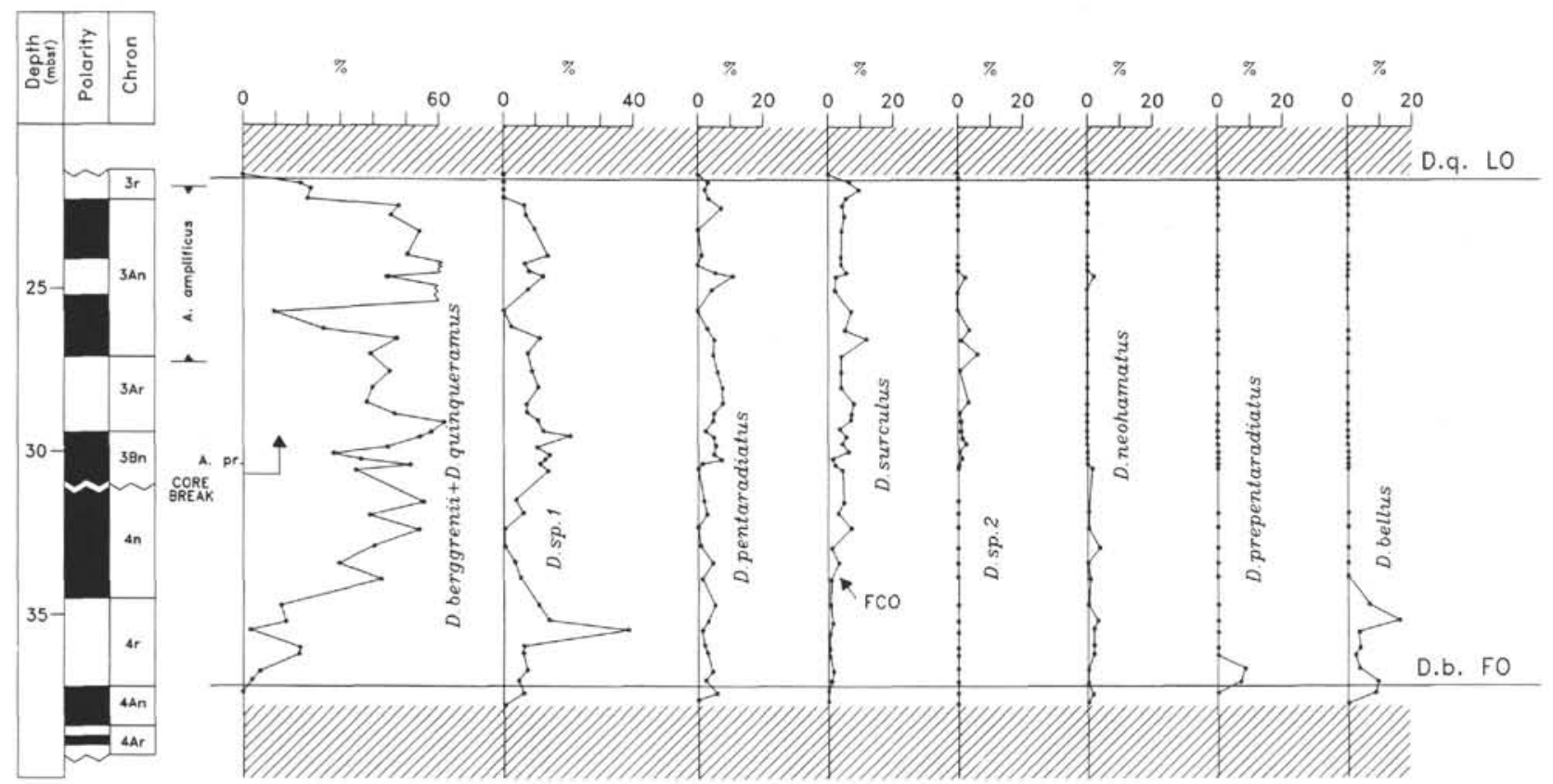

Figure 14. Abundance patterns of late Miocene selected calcareous nannofossils at Hole 711B. $(\mathrm{mbsf})=$ meters below seafloor. Notation as specified in Figure 3. D.q. = Discoaster quinqueramus; A.pr. = Amaurolithus primus; D.b. = Discoaster berggrenii . Magnetostratigraphy from Schneider and Kent (1990), revised.

quences (i.e., Site 848). Representatives of the genus Catinaster are very rare or absent at equatorial Sites 846,849 , and 850 (see Raffi and Flores, this volume), and this probably results from peculiar productivity conditions that controlled carbonate accumulation (nannofossil assemblages) at those sites.

The correlation of the FO at $C$. coalitus to the GPTS has been controversial and has significant bearing for correlating marine magnetic anomalies to the GPTS (see Berggren et al., 1985: 222).

Berggren et al. (1985) correlated the FO of $C$. coalitus to Subchron 5r.2r at Site 563, whereas Poore et al. (1983), at the mid-latitude South Atlantic, correlated the event to the lower part at Chron $5 n .2 n$.

We calibrated the event at Site 845 (Fig. 12), where it occurs in the lowermost part of Chron C5n.2n, in agreement with calibration in the mid-latitude South Atlantic (Poore et al., 1985; Hsü et al., 1984). A similar magnetostratigraphic position for the FO of $C$. coalitus can be inferred at mid-latitude Site 608. In fact, although seen at that site, $C$. coalitus is rare and sporadic in its lower range, and the lowermost specimens were observed at the base of Chron $5 n .2 n$ (Olafsson, 1991).

Notwithstanding the indications of an ecologic control on Catinaster distribution in different areas, our data indicate that the $\mathrm{FO}$ of C. coalitus can be a useful biostratigraphic marker in low-latitude sediments, as previously suggested by Bukry (1973).

\section{FO of Discoaster calcaris (16)}

One of the additional markers indicated by Bukry (1973) for characterizing CN6 and CN7 transition is Discoaster calcaris. Its distribution range was obtained at Sites 714,844 , and 845 (Figs. 8 , 11 , and 12). Our data agree with Bukry's indication of the FO of $D$. calcaris within the $C$. coalitus range (Zone $\mathrm{CN} 6$ ). In the tropical Indian Ocean (Site 714, Fig. 8), the species is particularly abundant and reaches high percentage values at some intervals $(>20 \%)$, while its range extends to Zone CN7. In the equatorial Pacific (Figs. 11 and 12 ), D. calcaris gives a similar and distinctive biostratigraphic signal, although it is less abundant and its range is restricted to a short interval within Zone CN6.

\section{LO of Discoaster exilis (17)}

Bukry (1973) suggested that the LO of Discoaster exilis is a useful guide for monitoring the upper part of the Catinaster coalitus Zone (CN6).

We have established the final range of D. exilis at Sites 714 (Fig. 8), 710 (Fig. 10), 845 (Fig. 12), and 848 (Fig. 13). In agreement with Bukry's suggestion, in the tropical Indian Ocean, the species disappears in the upper part of Zone CN6 at Site 714 (Fig. 8). At Site 710 (Fig. 10), it is not observed in Zone CN7. In contrast, in equatorial Pacific Ocean, D. exilis is present throughout Zone CN6, becoming extinct in the lowermost part of Zone CN7 (Figs. 12 and 13). Also, note the contrast in abundance observed between the successions retrieved at Sites 845 (Fig. 12) and 848 (Fig. 13). This indicates that local ecological conditions and biogeography seem to control the abundance and the final exit of the species, and $D$. exilis does not seem an accurate biohorizon. However, it may be useful to note that $D$. exilis survives only for a short time, in low abundance, and the appearance of Discoaster hamatus and other five-rayed discoasterids. Therefore, its exinction is a significant element of the major turnover in nannofossil assemblages at the base of Zone CN7, as evidenced by Bukry (1975), Rio et al. (1990a), and Raffi and Flores (this volume).

\section{FO of Discoaster bellus Group (18)}

The appearance of the distinctive Neogene discoasterids having five symmetrical rays (Discoaster bellus group) was considered to occur approximately together with the appearance of $D$. hamatus (base of Zone CN7) by Bukry (1973) and Rio et al. (1990a). As shown by data reported in Figures 8, 9, and 10, the two events are virtually coincident in the tropical Indian Ocean, whereas in the equatorial Pacific Ocean, the appearance of $D$. bellus occurs slightly below (Figs. 11,12,13). At mid-latitude Site 608, Gartner (1992) recorded a similar shift between $D$. bellus group FO and the $D$. hamatus FO. It is difficult to evaluate the reasons for the discrepancies between data from Indian ocean and data from the equatorial Pacific and North Atlantic oceans. These most probably result from vagaries in the stratigraphic record 


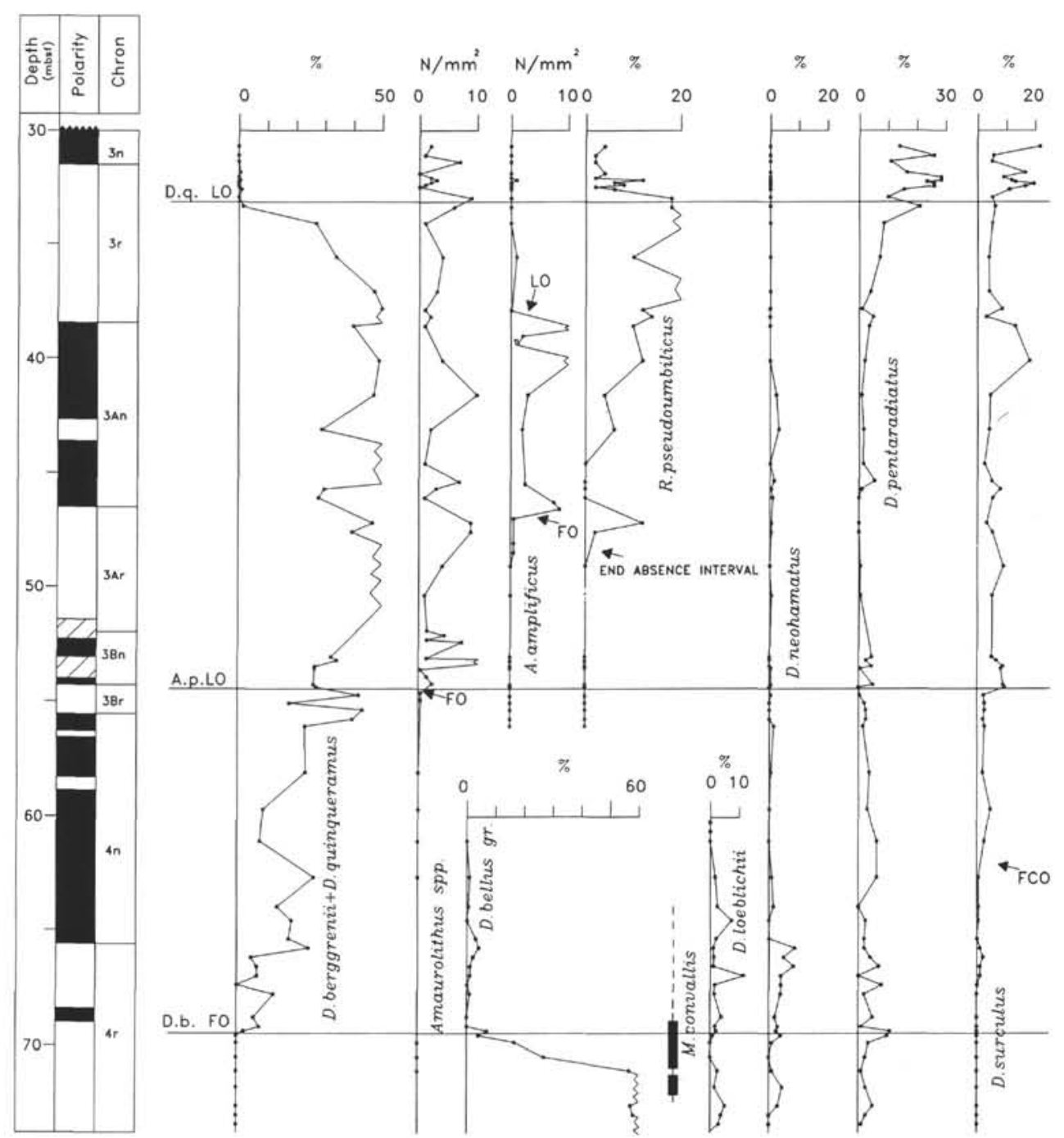

Figure 15. Abundance patterns of late Miocene selected calcareous nannofossils at Site $853 .(\mathrm{mcd})=$ meters composite depth. Notation as specified in Figure 3. D.q. = Discoaster quinqueramus; A.p. = Amaurolithus primus; D.b. = Discoaster berggrenii. Magnetostratigraphy from site chapters in Mayer, Pisias, Janecek, et al. (1992) and Schneider (this volume).

(i.e., slumping at Site 710 near the $D$. hamatus FO) or operational biases in the Indian Ocean sequences, rather than to real diachroneity. At Sites 844,845 , and 848 (Figs. 11, 12, and 13), the FO of D. bellus group occurs in the lower part of Subchron $5 \mathrm{n} .2 \mathrm{n}$. A similar chronostratigraphic position for the event was recorded at North Atlantic Site 608 (Gartner, 1992), indicating biochronologically consistency for the event between the two areas.

\section{LO of Coccolithus miopelagicus (19)}

Burky (1973) first suggested that the disappearance of Coccolithus miopelagicus within the Catinaster coalitus Zone (CN6) is a useful biostratigraphic guide. Olafsson (1991) recorded the LO of $C$. miopelagicus in the same stratigraphic position at the North Atlantic Site 608 and suggested the possibility of dividing Zone CN6 into two subzones. We have followed in detail the final range of $C$. miopelagicus at Sites 714 (Fig. 8), 844 (Fig. 11), 845 (Fig. 12), and 848 (Fig. 13). At all these sites, the LO of C. miopelagicus occurs within the range of $C$. coalitus, below the FO of $D$. hamatus and slightly above the FO of D. bellus group in the Pacific sites. The event is abrupt, and maintaining the same biostratigraphic position also at mid-latitude sequence of Site 94-608 (Fig. 17), it is considered as a reliable bio- stratigraphic event. At equatorial Pacific Sites 844 (Fig. 11), 845 (Fig. 12), and 848 (Fig. 13), the LO of C. miopelagicus occurs in the lower part of Subchron C5n. At mid-latitude Site 608, Olafsson (1991) and Gartner (1992) detected the event at the base of Subchron C5n. This indicates that some diachroneity may be inferred for the extinction of C. miopelagicus between low- and mid-latitude areas.

\section{FO of Catinaster calyculus (20)}

As reported in Bukry's zonation (1973), the upper part of Zone $\mathrm{CN} 7$ (D. hamatus range) is characterized by the presence of Catinaster calyculus, whose appearance defines the boundary between Subzones $\mathrm{CN} 7 \mathrm{a}$ and $\mathrm{CN} 7 \mathrm{~b}$. This biostratigraphic marker could not be used in the studied sequences, in both the equatorial Indian (Rio et al., 1990a) and Pacific oceans (Raffi and Flores, this volume), because in both areas C. calyculus appears in a lower stratigraphic level than indicated by Bukry (1973). The detailed distribution obtained at Sites 714 (Fig. 8), 844 (Fig. 11), and 845 (Fig. 12) show that the FO of $C$. calyculus occurs below the FO of $D$. hamatus (basal boundary of $\mathrm{CN} 7$ ). Moreover, the species is rare and scattered all along its range, and, therefore it is considered unsuitable as a biostratigraphic and biochronologic tool. 


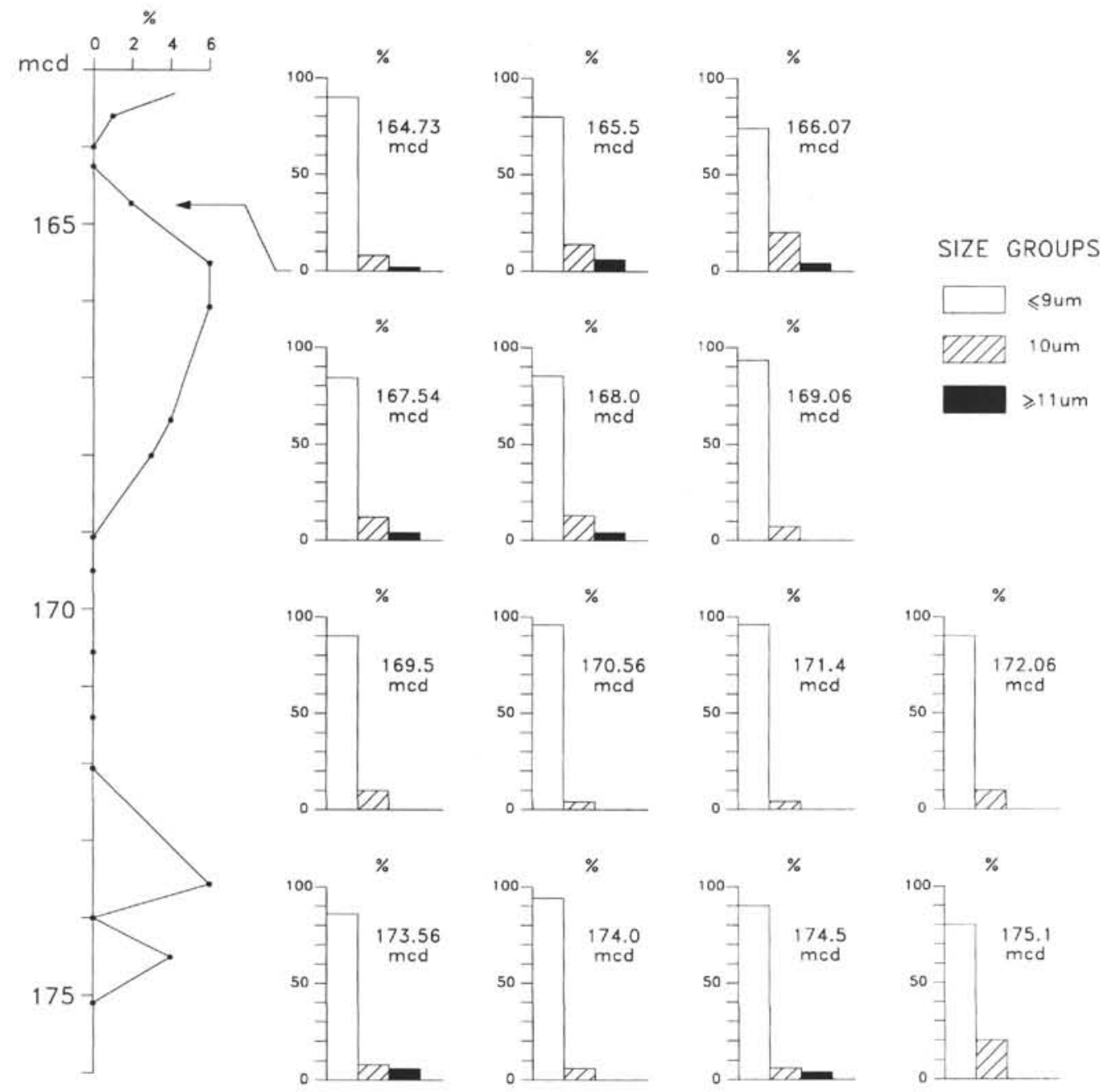

Figure 16. Distribution pattern of Calcidiscus macintyrei $(\geq 11 \mu \mathrm{m})$ and variation of size of Calcidiscus population within CN5b interval at ODP Site 845.

\section{FO of Discoaster hamatus (21)}

The FO of Discoaster hamatus is a zonal boundary event in both Bukry's (1973) and Martini's (1971) standard zonations, respectively, the $\mathrm{CN} 6 / \mathrm{CN} 7$ and NN8/NN9 boundaries.

The initial distribution pattern of $D$. hamatus was established at Indian Ocean Sites 714 (Fig. 8) and 710 (Fig. 10) and at Pacific Ocean Sites 844 (Fig. 11), 845 (Fig. 12), and 848 (Fig. 13). As observed for most of the discoasterid species, in the equatorial Indian Ocean sequences, we noted higher abundances of $D$. hamatus than in equatorial Pacific Ocean sediments. The species abundance reaches high percentage values relative to the other discoasterids $(\geq 20 \%)$ at Sites 709 and 714 , whereas $D$. hamatus is generally less common in the other sequences. However, the recognition of this FO event is relatively easy, whatever abundance and preservation conditions may be.

A sufficiently precise magnetostratigraphic position of the FO of D. hamatus was established at the Pacific Sites 845 and 848 (Figs. 12 and 13), where it occurs in the middle part of Subchron $5 n .2 n$. A similar position may be assumed from the data in Site 844 (Fig. 11), although the base of Subchron $5 \mathrm{n} .2 \mathrm{n}$ was not recognized at this site (Schneider et al., this volume).

At Indian Ocean Site 710 (Fig. 10), the lowermost observed specimens of $D$. hamatus were recorded in an interval disturbed by slumping, just below a normal polarity interval interpreted as Chron $5 \mathrm{n}$ (Schneider and Kent, 1990). At this site, we assumed that both the true FO of $D$. hamatus and the base of Chron $5 n$ have been obscured by slumping and displaced material, making unprecise the calibration of the event in this sequence.

In the North Atlantic Ocean, the FO of D. hamatus was calibrated at Sites 563 (Berggren et al., 1985; Miller et al., 1985) and 608 (Olafsson,1991; Gartner, 1992). At Site 563, the event is recorded in the lower part of Subchron $5 n .2 n$, apparently in a lower position from that we observed in the equatorial Pacific. At mid-latitude Site 608, Olafsson (1991) found that $D$. hamatus was very rare and sporadic in the lower part of its range and increased in abundance at the top of Chron $5 \mathrm{n}$. The first rare occurrence of the species was detected in the middle part of Subchron $5 \mathrm{n} .2 \mathrm{n}$, therefore, in a position similar to that observed in low-latitude sequences.

The comparison of distribution patterns of $D$. hamatus in sequences from different latitudes indicates that the FO of $D$. hamatus is a good (easily detected and sufficiently isochronous) event in lowlatitude sediments. However, it does not represent a useful and reliable correlation line between low- and mid-latitude sequences as Site 608 , where $D$. hamatus is very rare in the lower range and its true FO can be detected only in high-resolution sampling.

\section{LO of Catinaster coalitus (22) and Catinaster calyculus (23)}

Bukry (1973) reported the extinction of Catinaster coalitus and Catinaster calyculus as occurring above the LO of D. hamatus and below the FO of $D$. neorectus and D. loeblichii (Subzone CN8a). 
Data obtained at Indian Ocean Sites 714 and 710 (Figs. 8 and 10) and Pacific Site 844 (Fig. 11) did not confirm this statement of Bukry for the two LO events. At Site 844, the presence of barren samples in the upper part of Zone CN7 did not allow us to obtain detailed information about the Catinaster distibution pattern in that interval. Anyway, we did not record any specimens of Catinaster in the overlying Zone CN8. A similar result was obtained at Indian Ocean sites (Figs. 8 and 10 ), where $C$. coalitus and $C$. calyculus disappear in the lower and in the upper part of $\mathrm{CN} 7$, respectively. As already noted above, the distribution patterns of Catinaster spp. seem to be controlled by paleoecologic factors that can account for the discrepancies in reporting their biostratigraphic events.

\section{FO of Discoaster prepentaradiatus (24)}

Bukry (1973) reported the FO of Discoaster prepentaradiatus in Zone CN7. Detailed distribution patterns of this distinct species were never reported in the literature. Data collected in in the equatorial Pacific sequences did not provide sufficiently detailed information on the $D$. prepentaradiatus range in the area. For instance, at Site 844 (Fig. 11), the species occurs sporadically within the upper part of Zones CN7 and CN8. Its FO event could not be detected owing to the presence of dissolution intervals. On the contrary, in tropical Indian Ocean sequences $D$. prepentaradiatus was consistently recorded. At Sites 714 and 710 (Figs. 8 and 10), its FO was recorded above the $D$. hamatus $\mathrm{FO}$ (within Zone $\mathrm{CN} 7$ ). In the lower range, this discoasterid is rare and sporadic and increases in abundance just below the D. hamatus LO. The same distribution pattern was observed in the highly compressed Zone CN7 interval at Site 709 (Fig. 9).

Although the FO of $D$. prepentaradiatus is not useful for biostratigraphic correlations between the Pacific and Indian oceans, the species provides a good biostratigraphic signal for characterizing the transition between Zones $\mathrm{CN} 7$ and $\mathrm{CN} 8$. As regards the upper range of D. prepentaradiatus, the species' decline occurs within Subzone CN8b, as already noted by Bukry (1973), and the uppermost rare specimens are recorded just above the FO of $D$. berggrenii in both Pacific and Indian oceans.

\section{FO of Discoaster neohamatus (25)}

Bukry (1973) suggested that Discoaster neohamatus appears within the range of $D$. hamatus (Zone CN7). At Site 608, Gartner (1992) found the FO of D. neohamatus within Zone CN7, but noted that at this site the species is not an entirely reliable marker, being rare and sporadic.

We established the distribution pattern of $D$. neohamatus at Sites 714 (Fig. 8), 709 (Fig. 9), 710 (Fig. 10), 844 (Fig. 11), and 848 (Fig. 13). The species is better represented in the tropical Indian Ocean than in the equatorial Pacific Ocean. Moreover, its FO seems diachronous between the two areas. In fact, at Indian Ocean Sites 714, 709, and 710 (Figs. 8-10), D. neohamatus appears as rare above the FO of $D$. hamatus. At Site 710, the position of the event corresponds to midChron 5n. At Pacific Sites 844 and 848 (Figs. 11 and 13), the first rare D. neohamatus specimens were observed in a higher stratigraphic position, closer to the LO of $D$. hamatus and corresponding to the upper part of Chron $5 \mathrm{n}$. Note that in both the Indian and Pacific oceans, the increase in abundance of $D$. neohamatus seems to occur synchronously within Zone $\mathrm{CN} 8$, in the interval corresponding to Chrons $4 \mathrm{Ar}$ and $4 \mathrm{An}$.

\section{LO of Discoaster hamatus (26)}

The LO of Discoaster hamatus is a zonal boundary event in both Bukry's and Martini's standard zonations, respectively, the CN6/CN7 and NN8/NN9 boundaries.

We quantitatively determined this event in the upper Miocene of all the studied sequences except the Pacific Site 845 , where the stratigraphic interval corresponding approximately to Zones $\mathrm{CN} 7$ and CN8 (upper Chrons 5n and 4A) is barren of nannofossils (Fig. 12).

$D$. hamatus is generally rare or scarce and exhibits high abundances only at Site 714 (Fig. 8). The species is always easily recognized, even in overgrown assemblages. Its LO event is associated with Subchron $4 \mathrm{Ar} .2 \mathrm{r}$ as a result of correlation to magnetostratigraphic records of Sites 710 (Fig. 10), 844 (Fig. 11), and 848 (Fig. 13). This finding does not agree with the calibration obtained in the North Atlantic at Sites 563 (Miller et al., 1985) and 608 (Olafsson, 1991; Gartner, 1992). At Site 94-608, Olafsson (1991) recognized the LO of D. hamatus at the base of a normal polarity interval (Subchron $4 \mathrm{Ar} .2 \mathrm{n}$ ), whereas Gartner (1992) indicated a lower position, close to the $\mathrm{C} 5 \mathrm{n} / \mathrm{C} 4 \mathrm{Ar}$ boundary. The latter finding agrees with the data of Miller et al. (1985) at Site 563. Nevertheless, D. hamatus seems to have an earlier extinction in the mid-latitude North Atlantic Ocean than in the low-latitude Indian and Pacific oceans. Its LO event, therefore, is a reliable marker for correlation only in low-latitude oceanic sediments.

\section{FO of Minylitha convallis (27) and Its Distribution Range}

Bukry (1973) noted the appearance of Minylitha convallis within Subzone CN8a. This nannofossil occurs up to Subzone CN9a, but it is most typical of Zone CN8. We monitored the distribution pattern of this distinctive species at Sites 709 (Fig. 9), 710 (Fig. 10), and 714 (Fig. 8) in the Indian Ocean and at Sites 844 (Fig. 11), 845 (Fig. 12), and 848 (Fig. 13) in the equatorial Pacific Ocean.

At Indian Ocean sites, the FO of $M$. convallis approximates the LO of D. hamatus. The species is well represented and is a useful guide for recognizing the lower boundary of Zone CN8. Its extinction in the tropical Indian Ocean occurs well above the FO of $D$. berg: grenii, slightly below the FO of Amaurolithus primus (Figs. 9 and 10). At Site 710, the FO of $M$. convallis is associated with the Subchron C4Ar.2r, while its LO is associated with Subchron C4n.2n. At equatorial Pacific sites (Figs. 11-13), the species is poorly represented, probably because of paleoecologic restrictions (Raffi and Flores, this volume) and has a restricted range, compared with its occurrence in the tropical Indian Ocean (Fig. 17). At Site 845 (Fig. 12), the distribution pattern of $M$. convallis is incomplete for the presence of a dissolution interval in the sequence. At Sites 844 (Fig. 11) and 848 (Fig. 13), M. convallis is recorded in the interval from Subchron $4 \mathrm{Ar}$. In to Subchron $4 \mathrm{n} .2 \mathrm{n}$.

At North Atlantic Site 608, Gartner (1992) detected the FO and the LO of $M$. convallis in the upper part of Chron $5 \mathrm{n}$ and in the upper part of Chron $4 \mathrm{n}$, respectively, showing a somewhat expanded range with respect to the tropical Indian Ocean. From the available data, it is evident that while the $M$. convallis range is a useful guide for recognizing the time interval corresponding to $\mathrm{CN} 8$ and $\mathrm{CN} 9 \mathrm{~b}$ time, its FO and LO events are unreliable markers for long distance correlations.

\section{LO of Discoaster bollii (28)}

At North Atlantic Site 608, Gartner (1992) recorded the LO of Discoaster bollii above the LO of D. hamatus and correlated it to GPTS as occurring in mid-Chron 4Ar. He considered this event unreliable because the species was very rare and occurred sporadically at that mid-latitude site. Our data on D. bollii distribution from the equatorial Indian and Pacific oceans indicate a similar stratigraphic position for its LO event. At Indian Ocean Site 710 (Fig. 10) and Pacific Site 844 (Fig. 11), D. bollii becomes extinct just above the LO of $D$. hamatus, whereas at Site 714 it disappears just below the LO of D. hamatus (Fig. 8). The event is easily detected because the species is well represented in the underlying intervals and declines abruptly before the extinction. At Site 845 (Fig. 12) and 848 (Fig. 13), problems of preservation (severe dissolution at the former and strong overgrowth at the latter site) hampered the definition of $D$. bollii distribution and the recognition of the LO event. 
Correlation to magnetostratigraphic records at Sites 844 and 710 shows that the LO of D. bollii is associated with Subchron 4Ar.2r. This result is in agreement with the data from North Atlantic Sites 563 (Miller et al., 1985) and 608 (Gartner, 1992) (Fig. 17).

\section{FO of Discoaster loeblichii (29) and the FO of Discoaster neorectus (30)}

These two events were used by Bukry (1973) to divide Zone CN8 into two subzones (CN8a and $\mathrm{CN} 8 \mathrm{~b}$ ). In the tropical Indian Ocean (Leg 115 sections), Discoaster loeblichii and Discoaster neorectus do not give any biostratigraphic signal, being rare and sporadic (Rio et al., 1990a). In the equatorial Pacific, $D$. neorectus is very rare as well, whereas $D$. loeblichii was recorded consistently (see Raffi and Flores, this volume). The FO of D. loeblichii was calibrated at Site 844 (Fig. 11) as occurring in the lower part of Chron 4r, below the FO of $D$. berggrenii. D. loeblichii is discontinuously distributed upward and becomes extinct within Subzone CN9a, in an interval corresponding to upper Chron $4 \mathrm{n} /$ lower Chron $3 \mathrm{Bn}$. This is in agreement with data from mid-latitude North Atlantic Site 608, where Gartner (1992) recognized the FO and LO of D. loeblichii in similar magnetostratigraphic positions (Fig. 17).

\section{Absence Interval of Reticulofenestra pseudoumbilicus}

At Indian Ocean Leg 115 sequences, in the stratigraphic interval encompassing most of Zones CN8 and CN9, Rio et al. (1990a) recorded the almost total disappearance of large specimens $(>7 \mu \mathrm{m})$ of Reticulofenestra pseudoumbilicus (R. pseudoumbilicus Paracme). A similar absence interval, in the same stratigraphic position, was observed at the equatorial Pacific sequences of Leg 138 (Raffi and Flores, this volume). In both areas, the interval begins with the total disappearance of all the representatives of the genus Reticulofenestra, both small- and large-sized species. The smaller forms (ascribed to $R$. haqi, $R$. minuta and $R$. minutula) reenter slightly above, whereas $R$. pseudoumbilicus is missing, for a long stratigraphic interval. Correlation to magnetostratigraphy indicates an extension from the upper part of Chron $4 \mathrm{An}$ to the lower part of Chron $3 \mathrm{Ar}$ (Fig. 17). The data obtained in the equatorial Pacific point to a wider geographic extent of this stratigraphic feature, which is clearly correlatable between tropical/equatorial regions. Note that a similar turnover in the placolith assemblage was observed at North Atlantic Site 608 by Gartner (1992) in the same stratigreaphic level (around the C4An/C4r boundary) and was interpreted as a major change in productivity.

\section{FO of Discoaster pentaradiatus (31)}

Discoaster pentaradiatus is known to be a major component of the discoasterid assemblage of the Pliocene. It enters the stratigraphic record in the late Miocene, in the time interval corresponding to Zone CN8 (Bukry, 1973). Data obtained in this study partially agree with the known distribution range. In the equatorial Pacific Site 844 (Fig. $11), D$. pentaradiatus is present as rare and scattered, starting from Zone $\mathrm{CN} 8$, associated with the upper part of Chron $4 \mathrm{Ar}$, whereas at Site 848 (Fig. 13), it was found only in spot samples in the upper Miocene interval.

Data from the tropical Indian Ocean differ slightly from results of Site 844. At Sites 714 (Fig. 8) and 710 (Fig. 10), rare D. pentaradiatus specimens were detected within the upper part of Zone CN7. Furthermore, the distribution pattern of $D$. pentaradiatus in the tropical Indian Ocean (Figs. 9, 10, and 14) shows generally higher abundances than those in the equatorial Pacific sequences (Figs. 11, 12, 13, and 15).

A magnetostratigraphic position for the $\mathrm{FO}$ of $D$. pentaradiatus similar to that obtained at mid-latitude Site 608 (Gartner, 1992) was detected in the equatorial Pacific (Fig. 17). The event is not easily detectable, since $D$. pentaradiatus is rare and discontinuous all along its lower range and is not useful as a precise biostratigraphic marker for the late Miocene. Its distribution becomes more continuous within Subzone CN9a, above the appearance of $D$. berggrenii and $D$. quinqueramus, in all the studied sequences except Site 848 . The gradual increase in abundance of $D$. pentaradiatus occurs concomitantly with the decline and/or disappearance of other discoasterids, such as the $D$. bellus group, D. loeblichii, and the last representatives of D. neohamatus.

\section{FO of Discoaster berggrenii (32)}

Although we have quantitatively evaluated Discoaster berggrenii and Discoaster quinqueramus as a single taxonomic unit (see Raffi and Flores, this volume), we considered and defined, following Bukry (1973), the appearance event of the species D. berggrenii, which corresponds to the biostratigraphic boundary $\mathrm{CN} 8 / \mathrm{CN} 9$.

In both Leg 115 sequences of the tropical Indian Ocean (Figs. 9, 10, and 14) and Leg 138 sequences of the equatorial Pacific (Figs. 11, 12,13 , and 15 ), the FO of $D$. berggrenii occurs in a magnetic reversed polarity interval interpreted as lowermost Chron 4r. D. berggrenii enters the stratigraphic record with few specimens, and low abundances characterize the lower part of its range. This feature, commonly observed in the distribution patterns of nannofossils, indicates that "first occurrence" events never appear as abrupt events when detected in a high-resolution sampling set (the "morphologies" of many FO events of nannofossils are similar).

D. berggrenii becomes a major element of the discoasterid assemblage close to the base of Chron $4 \mathrm{~N}$, when also the D. quinqueramustype specimens are consistently present. Note that the spreading of the $D$. berggrenii- $D$. quinqueramus component coincides with the decline and subsequent disappearance of $D$. bellus group representatives in the discoasterid assemblage.

Our calibration of $D$. berggrenii FO differs from the calibration reported in Berggren et al. (1985) (data from Haq et al.,1980, and Miller et al.,1985), who correlated the $D$. quinqueramus $(=D$. berggrenii) FO with the upper part of Chron 4An. It also differs from $D$. quinqueramus FO datum reported in Backman et al. (1990) as occurring close to the base of Chron $4 \mathrm{n}$, because they defined and calibrated the appearance event of $D$. quinqueramus-type specimens.

Data from north Atlantic Site 608 (Gartner, 1992) indicate that the FO of $D$. berggrenii occurs in the upper part of Chron $4 \mathrm{n}$. This diachroneity with our data from low-latitude sequences presumably reflects a paleoceanographic control on distribution of $D$. berggrenii and $D$. quinqueramus at this mid-latitude site, where the two discoasterids are present discontinuously in very low abundances (Gartner, 1992: Fig. 1).

\section{FCO of Discoaster surculus (33)}

Bukry (1973) stated that Discoaster surculus appears as sporadic in the upper part of Zone CN8 (below the FO of D. berggrenii), probably developing from $D$. pseudovariabilis. It becomes more commonly and continuously distributed upward, within Zone CN9, with more typical specimens. Specifically, the appearance of typical D. surculus provides an alternative mean for recognizing the base of Zone CN9 besides FO of $D$. berggrenii. We have monitored the distribution of D. surculus in the studied sequences (Figs. 9-15) and partially confirmed Bukry's observations, as regards its lowest sporadic and discontinuous occurrences within Zone $\mathrm{CN} 8$. Both in tropical Indian Ocean (Figs. 9, 10, and 14) and equatorial Pacific Sites (Figs. 11, 12,13, and 15),D. surculus is continuously present starting from Zone CN9, just above the appearance level of D. berggrenii. We recognized its FCO in the lower part of Zone CN9, at an interval corresponding to middle Chron 4 n.2n (Figs. 9-15 and 17). This calibration results in an older than previous calibration of $D$. surculus FO obtained by Haq et al. (1980) in the Pacific, and Mazzei et al. (1979) in the Atlantic in the lower part of Chron 3Br (Epoch 6). The 
discrepancy is most probably the result of the weakness of this datum event, whose traceability depends on subjective taxonomic concept, methodological methods, and environmental control. Anyhow, we could consistently correlate the FCO of $D$. surculus between the low-latitudes of the Indian and Pacific oceans. This FCO seems to be slightly diachronous with the event recorded at mid-latitude North Atlantic Site 608 (Fig. 17), where Gartner (1992) calibrated it at the very top of Chron 4.

\section{FO of Amaurolithus primus (34)}

The appearance of the horseshoe-shaped nannofossil Amaurolithus primus has been used by Bukry to define the boundary of Subzones CN9a and CN9b. Available calibrations of the FO of $A$. primus, previously obtained in different areas, show some variability. This could be the result of different analytical methods applied for detecting the event (namely "variable" accuracy and time spent in looking the microscope). Specimens belonging to the ceratolithid group are generally present in low abundance in the nannofossil assemblages. Moreover, in the earliest forms, the typical crescentshaped outline is not clearly evident, with these primitive ceratolithids being more robust and having a thickened arch. Therefore, the precision in locating the $\mathrm{FO}$ event of $A$. primus can be affected by variable analytical accuracy.

We compared the data obtained at tropical Indian Ocean Site 710 (Fig. 10, and Rio et al., 1990) with data from the equatorial Pacific Sites $844,845,848$, and 853 (Figs. 11-13 and 15). The appearance datum of $A$. primus is a useful biostratigraphic event that seems isochronous in the two areas, occurring within Chron $3 \mathrm{Br}$ (Fig. 17). Note that the distribution patterns of Amaurolithus representatives are similar in the two areas and show similar variations in abundance and discontinuous distribution in some intervals. At Site 845, the distribution of Amaurolithus spp. is clearly affected by poor preservation conditions (dissolution and dilution) in the interval.

As regards comparison with the aforementioned previous calibrations of A. primus FO, in the equatorial Pacific Haq et al. (1980) calibrated the FOs of $A$. primus and A. delicatus as occurring in the normal polarity event of Epoch 6 (Chron $3 \mathrm{Bn}$ ). They probably failed to detect the earliest specimens of $A$. primus, as demonstrated by the cooccurrence of $A$. primus and $A$. delicatus (the latter species appears above the former, when the ceratolithids evolve morphologically toward more delicate forms, see Raffi and Flores, this volume). Data from Site 608 (Gartner, 1992) indicate for A. primus FO event a calibration that is probably slightly diachronous to that obtained at lowlatitude Indian and Pacific ocean sediments, although the polarity record at Site 608 is not precise in the interval corresponding to Chron $3 \mathrm{Br}$.

The data obtained indicate that the FO of A. primus is a reliable biostratigraphic event in low-latitude oceanic environments, isochronous between equatorial Indian and Pacific oceans.

\section{FO and LO of Amaurolithus amplificus $(35,36)$}

Amaurolithus amplificus (synonym of Ceratolithus dentatus of Bukry, 1973) is an easily recognized, short-ranged taxon, which appears and becomes extinct within the stratigraphic interval above the FO of A. primus and below the LO of D. quinqueramus (Subzone $\mathrm{CN} 9 \mathrm{~b}$ ).

The usefulness as biostratigraphic marker of A. amplificus has been pointed out for the equatorial Indian (Rio et al., 1990a) and Pacific (Raffi and Flores, this volume) oceans. In the two areas, the FO and LO of A. amplificus consistently correlate with the base and the top of Chron C3An, respectively. This was observed at Sites 710 and 711 in the Indian Ocean (Fig. 17; see also Figs. 7, 8, and 14 in Rio et al., 1990a) and at Sites 844, 845, and 853 in the Pacific Ocean (Figs.11, 12, 15, and 17).

Haq et al. (1980), as well, associated the range of A. amplificus to Chron $3 \mathrm{An}$ in piston cores from the Pacific Ocean, even if they reported a somewhat shorter range for the species. No detailed data are available from mid-latitude areas.

Although this ceratolithid generally is present in low abundance, its distribution range can be easily defined through sufficiently accurate analyses. Therefore, the LO and FO of A. amplificus can be considered reliable biostratigraphic events that provide two excellent correlation lines within and between the equatorial Indian and Pacific oceans. The two events can be used to increase biostratigraphic and chronostratigraphic resolution in the late Miocene (Raffi and Flores, this volume).

\section{LO of Discoaster quinqueramus (37)}

Discoaster quinqueramus is a major component of the discoasterid assemblages in both the tropical Indian (Figs. 9, 10,14) and Pacific oceans (Figs. 11, 12, 13, and 15). Its extinction occurs just above the LO of A. amplificus and below the FO of $C$. acutus in all the investigated sequences (Fig. 17) (see Raffi and Flores, this volume, and Rio et al., 1990a) and is an abrupt event. Therefore, it is an excellent biohorizon and correlation tool within and between the two equatorial regions.

The LO of $D$. quinqueramus can be precisely calibrated to the GPTS at Site 710 in the Indian Ocean (Fig. 10) and at Sites 844, 845, and 853 in the Pacific Ocean (Figs. 11, 12, and 15). In both areas, it occurs in the mid-part of Chron $3 \mathrm{r}$, appearing as synchronous. Berggren et al. (1985) associated this event with the upper part of Chron 3An, referring to the questionable data of Mazzei et al. (1979) and Gartner (1973) (see Backman et al., 1990).

Our calibration of $D$. quinqueramus LO agrees with the calibration at mid-latitude North Pacific Site 577 (Monechi, 1985; Bleil, 1985; see also Gartner et al., 1984; Muza et al., 1987).

\section{CONCLUSIONS}

We have established the quantitative distribution patterns of 29 index calcareous nannofossils in the middle and upper Miocene sediments cored from the equatorial Pacific and tropical Indian oceans.

Our goal was to test the reliability of the classic biohorizons used in the standard zonations of Martini (1971) and Bukry (1973) and, possibly, to improve biostratigraphic resolution, which is low if compared to Pliocene and Pleistocene. Distinctness of the event and its correlatability among the different sections in the two low-latitude areas have been the critical factors in evaluating biostratigraphic reliability. The degree of synchroneity, as inferred by comparison with available magnetostratigraphies, is another critical factor.

In a time interval of about $8 \mathrm{~m} . \mathrm{y}$., from the LO of $S$. heteromorphus $(\approx 13.6 \mathrm{Ma}$ ) to the LO of D. quinqueramus $(\approx 5.5 \mathrm{Ma})$, a total of 37 events were investigated, both the conventional and some additional markers proposed in the literature. The standard zonations of Martini and Bukry provide a sound biostratigraphic framework for classifying and correlating sediments from the two considered areas. Specifically, the following events utilized as primary zonal definitions appear as reliable:

1. Sphenolithus heteromorphus LO,

2. Catinaster coalitus FO,

3. Discoaster hamatus FO,

4. Discoaster hamatus LO,

5. Discoaster berggrenii FO,

6. Amaurolithus primus $\mathrm{FO}$, and

7. Discoaster quinqueramus LO.

The FO of Discoaster kugleri (CN5a/CN5b or NN6/NN7 boundary) is a weak datum, with the species being very rare and discontinuously distributed in its lower range. The first common and continuous occurrence (FCO) of D. kugleri appears more reliable than its true first occurrence.

Some events proposed by Bukry (1973) as primary definitions of subzonal boundaries have proved difficult to recognize. Namely, the 
FO of $C$. calyculus occurs in both areas within Zone CN6, and not in Zone CN7. D. loeblichii, whose FO defines the boundary CN8a/ CN8b together with $D$. neorectus FO, is virtually missing in the Indian Ocean. The marker $D$. neorectus is found scattered in both Indian and Pacific areas.

Other events that proved to be sound biostratigraphic correlation lines between the two equatorial areas are the following:

1. Cyclicargolithus floridanus LCO,

2. Triquetrorhabdulus rugosus $\mathrm{FCO}$,

3. Calcidiscus premacintyrei $\mathrm{LO}$,

4. Coronocyclus nitescens LO,

5. Discoaster kugleri FCO,

6. D. kugleri LCO,

7. Coccolithus miopelagicus LO,

8. Discoaster neohamatus FO,

9. Discoaster surculus FCO,

10. Amaurolithus amplificus FO, and

11. Amaurolithus amplificus LO.

Thus, we are provided with at least 18 distinct biostratigraphic correlation lines in the considered interval of about $8 \mathrm{~m}$.y. The average resolution obtainable is on the order of about 0.5 m.y.

All the aforementioned events were tied to the GPTS, thus providing a biomagnetostratigraphic framework for low-latitude areas. This integrated framework can be useful for evaluating diachroneity with sediments from other latitudes, when further data will be available. For the time being, we compared our data with biomagnetostratigraphic data from mid-latitude Site 608 (Olafsson, 1991; Gartner, 1992) (Fig. 17) and obtained new informations on the biostratigraphic and biochronologic reliability of the investigated events over geographic distance.

We did not provide accurate absolute-age calibrations for the events in this phase of our work. However, age evaluations for the events (Fig. 17), as recorded in eastern equatorial Pacific sequences of Leg 138, are reported in Raffi and Flores (this volume) and Shackleton et al. (this volume).

\section{ACKNOWLEDGMENTS}

We would like to thank L. Beaufort and G. Olafsson for their critical reviews. Funding for this study was provided by CNR Grant AI91.00913.05 to I. Raffi, CNR-Centro di Studio per la Geodinamica Alpina, Padova, and MPI Grant $40 \%$ to F. Proto Decima.

\section{REFERENCES}

Aubry, M.-P., 1984. Handbook of Cenozoic Calcareous Nannoplankton. Book 1: Ortholithae (Discoasters): New York (Micropaleontology Press).

, 1988. Handbook of Calcareous Nannoplankton (Book 2): Ortholithae (Catinasters, Ceratoliths, Rhabdoliths): New York (Micropaleontology Press).

, 1989. Handbook of Cenozoic Calcareous Nannoplankton (Book 3): Ortholithae (Pentaliths and Others). Heliolithae (Fasciculiths, Sphenoliths, and Others): New York (Micropaleontology Press).

1990. Handbook of Cenozoic Calcareous Nannoplankton (Book

4): Heliolithae (Helicoliths, Cribriliths, Lopadoliths, and Others): New York (Micropaleontology Press).

Backman, J., Schneider, D.A., Rio, D., and Okada, H., 1990. Neogene lowlatitude magnetostratigraphy from Site 710 and revised age estimates of Miocene nannofossil datum events. In Duncan, R.A., Backman, J., Peterson, L.C., et al., Proc. ODP, Sci. Results, 115: College Station, TX (Ocean Drilling Program), 271-276.

Backman, J., and Shackleton, N.J., 1983. Quantitative biochronology of Pliocene and early Pleistocene calcareous nannofossils from the Atlantic, Indian and Pacific oceans. Mar. Micropaleontol., 8:141-170.

- Abbreviations for names of organizations and publication titles in ODP reference lists follow the style given in Chemical Abstracts Service Source Index (published by American Chemical Society).
Barron, J.A., Keller, G., and Dunn, D.A., 1985. A multiple microfossil biochronology for the Miocene. In Kennett, J.P. (Ed.), The Miocene Ocean: Paleoceanography and Biogeography. Mem.-Geol. Soc. Am., 163:21-36.

Berggren, W.A., Kent, D.V., and Van Couvering, J.A., 1985. The Neogene: Part 2. Neogene geochronology and chronostratigraphy. In Snelling, N.J. (Ed.), The Chronology of the Geological Record. Geol. Soc. London Mem., 10:211-260.

Bleil, U., 1985. The magnetostratigraphy of northwest Pacific sediments, Deep Sea Drilling Project Leg 86. In Heath, G.R., Burckle, L.H., et al., Init. Repts. DSDP, 86: Washington (U.S. Govt. Printing Office), 441-458.

Bralower, T.J., Monechi, S., and Thierstein, H.R., 1989. Calcareous nannofossil zonation of the Jurassic-Cretaceous boundary interval and correlation with the geomagnetic polarity timescale. Mar. Micropaleontol., 14:153-235.

Bukry, D., 1971. Discoaster evolutionary trends. Micropaleontology, 17:43-52. 1973. Low-latitude coccolith biostratigraphic zonation. In Edgar, N.T., Saunders, J.B., et al., Init. Repts. DSDP, 15: Washington (U.S. Govt. Printing Office), 685-703.

1975. Coccolith and silicoflagellate stratigraphy, northwestern Pacific Ocean, Deep Sea Drilling Project Leg 32. In Larson, R.L., Moberly, R., et al., Init. Repts. DSDP, 32: Washington (U.S. Govt. Printing Office), 677-701.

- , 1978. Biostratigraphy of Cenozoic marine sediment by calcareous nannofossils. Micropaleontology, 24:44-60.

1985. Mid-Atlantic Ridge coccolith and silicoflagellate biostratigraphy, Deep Sea Drilling Project Sites 558 and 563. $\ln$ Bougault, H., Cande, S.C., et al., Init. Repts. DSDP, 82: Washington (U.S. Govt. Printing Office), 591-603.

Cande, S.C., and Kent, D.V., 1992. A new geomagnetic polarity time scale for the Late Cretaceous and Cenozoic. J. Geophys. Res., 97:13917-13951.

Clement, B.M., and Robinson, F., 1987. The magnetostratigraphy of Leg 94 sediments. In Ruddiman, W.F., Kidd, R.B., Thomas, E., et al., Init. Repts. DSDP, 94 (Pt. 2): Washington (U.S. Govt. Printing Office), 635-650.

Ellis, H.C., 1982. Calcareous nannoplankton biostratigraphy — Deep Sea Drilling Project Leg 60. In Hussong, D.M., Uyeda, S., et al., Init. Repts. DSDP, 60: Washington (U.S. Govt. Printing Office), 507-535.

Fornaciari, E., Backman, J., and Rio, D., 1993. Quantitative distribution patterns of selected lower to middle Miocene calcareous nannofossils from the Ontong Java Plateau. In Berger, W.H., Kroenke, L.W., Mayer, L.A., et al., Proc. ODP, Sci. Results, 130: College Station, TX (Ocean Drilling Program), 245-256.

Fornaciari, E., Raffi, I., Rio, D., Villa, G., Backman, J., and Olafsson, G., 1990. Quantitative distribution patterns of Oligocene and Miocene calcareous nannofossils from the western equatorial Indian Ocean. In Duncan, R.A., Backman, J., Peterson, L.C., et al., Proc. ODP, Sci. Results, 115: College Station, TX (Ocean Drilling Program), 237-254.

Gartner, S., 1992. Miocene nannofossil chronology in the North Atlantic, DSDP Site 608. Mar. Micropaleontol., 18:307-331.

Gartner, S., and Bukry, D., 1975. Morphology and phylogeny of the coccolithophycean family Ceratolithaceae. J. Res. U.S. Geol. Surv., 3:451-465.

Gartner, S., and Chow, J., 1985. Calcareous nannofossil biostratigraphy, Deep Sea Drilling Project Leg 85, eastern equatorial Pacific. In Mayer, L., Theyer, F., Thomas, E., et al., Init. Repts. DSDP, 85: Washington (U.S. Govt. Printing Office), 609-619.

Gradstein, F.M., Agterberg, F.P., Brower, J.C., and Schwarzacher, W.S., 1985. Quantitative Stratigraphy: Dordrecht (Reidel).

Hagelberg, T., Shackleton, N., Pisias, N., and Shipboard Scientific Party, 1992. Development of composite depth sections for Sites 844 through 854. In Mayer, L., Pisias, N., Janecek, T., et al., Proc. ODP, Init. Repts., 138 (Pt. 1): College Station, TX (Ocean Drilling Program), 79-85.

Haq, B.U., 1980. Biogeographic history of Miocene calcareous nannoplankton and paleoceanography of the Atlantic Ocean. Micropaleontology, 26:414443.

Hilgen, F.J., 1991. Astronomical calibration of Gauss to Matuyama sapropels in the Mediterranean and implication for the Geomagnetic Polarity Time Scale. Earth Planet. Sci. Lett., 104:226-244.

Loeblich, A.R., and Tappan, H., 1966. Annotated index and bibliography of the calcareous nannoplankton. Phycologia, 5:81-215.

1968. Annotated index and bibliography of the calcareous nannoplankton II. J. Paleontol., 42:584-598.

, 1969. Annotated index and bibliography of the calcareous nannoplankton III. J. Paleontol., 43:568-588.

- 1970a. Annotated index and bibliography of the calcareous nannoplankton IV. J. Paleontol., 44:558-574.

, $1970 \mathrm{~b}$. Annotated index and bibliography of the calcareous nannoplankton V. Phycologia, 9:157-174. 
1971. Annotated index and bibliography of the calcareous nannoplankton VI. Phycologia, 10:315-339.

-, 1973. Annotated index and bibliography of the calcareous nannoplankton VII. J. Paleontol., 47:715-759.

Martini, E., 1971. Standard Tertiary and Quaternary calcareous nannoplankton zonation. In Farinacci, A. (Ed.), Proc. 2nd Int. Conf. Planktonic Microfossils Roma: Rome (Ed. Tecnosci.), 2:739-785.

Mayer, L., Pisias, N., Janecek, T., et al., 1992. Proc. ODP, Init. Repts., 138 (Pts. 1 and 2): College Station, TX (Ocean Drilling Program).

Mazzei, R., Raffi, I., Rio, D., Hamilton, N., and Cita, M.B., 1979. Calibration of late Neogene calcareous plankton datum planes with the paleomagnetic record of Site 397 and correlation with Moroccan and Mediterranean sections. In von Rad, U., Ryan, W.B.F., et al., Init. Repts. DSDP, 47 (Pt. 1): Washington (U.S. Govt. Printing Office), 375-389.

Miller, K.G., Aubry, M.-P., Khan, K.J., Melillo, A.J., Kent, D.V., and Berggren, W.A., 1985. Oligocene-Miocene biostratigraphy, magnetostratigraphy and isotopic stratigraphy of the western North Atlantic. Geology, 13:257-261.

Miller, K.G., Feigenson, M.D., Wright, J.D., and Clement, B.M., 1991a. Miocene isotope reference section, Deep Sea Drilling Project Site 608: an evaluation of isotope and biostratigraphic resolution. Paleoceanography, 6:33-52

Miller, K.G., Wright, J.D., and Fairbanks, R.G., 199lb. Unlocking the Ice House: Oligocene-Miocene oxygen isotopes, eustasy, and margin erosion. J. Geophys. Res., 96:6829-6848.

Monechi, S., 1985. Campanian to Pleistocene calcareous nannofossil stratigraphy from the northwest Pacific Ocean, Deep Sea Drilling Project Leg 86. In Heath, G.R., Burckle, L.H., et al., Init. Repts. DSDP, 86: Washington (U.S. Govt. Printing Office), 301-336.

Muza, J.P., Wise, S.W., Jr., and Mitchener Covington, J., 1987. Neogene calcareous nannofossils from Deep Sea Drilling Project Site 603, Lower Continental Rise, western North Atlantic: biostratigraphy and correlations with magnetic and seismic stratigraphy. In van Hinte, J.E., Wise, S.W., Jr. et al., Init. Repts. DSDP, 93, Pt. 2: Washington (U.S. Govt. Printing Office), 593-616.

Okada, H., and Bukry, D., 1980. Supplementary modification and introduction of code numbers to the low-latitude coccolith biostratigraphic zonation (Bukry, 1973; 1975). Mar. Micropaleontol., 5:321-325.

Olafsson, G., 1989. Quantitative calcareous nannofossil biostratigraphy of upper Oligocene to middle Miocene sediment from ODP Hole 667A and middle Miocene sediment from DSDP Site 574. In Ruddiman, W., Sarnthein, M., et al., Proc. ODP, Sci. Results, 108: College Station, TX (Ocean Drilling Program), 9-22.

1991. Quantitative calcareous nannofossil biostratigraphy and biochronology of early through late Miocene sediments from DSDP Hole 608. Medd. Stockholms Univ. Inst. Geol. Geok., 203.

Parker, M.E., Clark, M., and Wise, S.W., Jr., 1985. Calcareous nannofossils of Deep Sea Drilling Project Sites 558 and 563, North Atlantic Ocean: biostratigraphy and the distribution of Oligocene braarudosphaerids. In Bougault, H., Cande, S.C., et al., Init. Repts. DSDP, 82: Washington (U.S. Govt. Printing Office), 559-589.

Perch-Nielsen, K., 1985. Cenozoic calcareous nannofossils. In Bolli, H.M., Saunders, J.B., and Perch-Nielsen, K. (Eds.), Plankton Stratigraphy: Cambridge (Cambridge Univ. Press), 427-554.

Prothero, D.R., 1990. Interpreting the Stratigraphic Record: New York (W.H Freeman).

Raffi, I., Backman, J., Rio, D., and Shackleton, N.J., 1993. Plio-Pleistocene nannofossil biostratigraphy and calibration to oxygen isotopes stratigraphies from Deep Sea Drilling Project Site 607 and Ocean Drilling Program Site 677. Paleoceanography, 8:387-408.

Raffi, I., and Rio, D., 1979. Calcareous nannofossil biostratigraphy of DSDP Site 132-Leg 13 (Tyrrhenian Sea-Western Mediterranean). Riv. Ital. Paleontol. Stratigr., 85:127-172.

Rio, D., Fornaciari, E., and Raffi, I., 1990a. Late Oligocene through early Pleistocene calcareous nannofossils from western equatorial Indian Ocean (Leg 115). In Duncan, R.A., Backman, J., Peterson, L.C., et al., Proc. ODP, Sci. Results, 115: College Station, TX (Ocean Drilling Program), 175-235.

Rio, D., Raffi, I., and Villa, G., 1990b. Pliocene-Pleistocene calcareous nannofossil distribution patterns in the Western Mediterranean. In Kastens, K.A. Mascle, J., et al., Proc. ODP, Sci. Results, 107: College Station, TX (Ocean Drilling Program), 513-533.

Shipboard Scientific Party, 1987. Site 608. In Ruddiman, W.F., Kidd, R.B. Thomas, E., et al., Init. Repts. DSDP, 94 (Pt. 1): Washington (U.S. Govt. Printing Office), 149-246.

Takayama, T., and Sato, T., 1987. Coccolith biostratigraphy of the North Atlantic Ocean, Deep Sea Drilling Project Leg 94. In Ruddiman, W.F.,
Kidd, R.B., Thomas, E., et al., Init. Repts. DSDP, 94 (Pt. 2): Washington (U.S. Govt. Printing Office), 651-702.

Theodoridis, S., 1984. Calcareous nannofossil biozonation of the Miocene and revision of the helicoliths and discoasters. Utrecht Micropaleontol. Bull., 32.

von Salis, A.K., 1984. Miocene calcareous nannofossil biostratigraphy of Deep Sea Drilling Project Hole 521A, Southeast Atlantic. In Hsü, K.J., LaBrecue, J.L., et al., Init. Repts. DSDP, 73: Washington (U.S. Govt. Printing Office), 425-427.

Young, J.R., 1990. Size variation of Neogene Reticulofenestra coccoliths from Indian Ocean DSDP cores. J. Micropaleontol., 9:71-85.

\section{Date of initial receipt: 18 June 1993 \\ Date of acceptance: 31 December 1993 \\ Ms 138SR-125}

\section{APPENDIX}

Calcareous Nannofossils Considered in this Chapter

(in alphabetic order of generic epithets)

Amaurolithus amplificus (Bukry and Percival, 1971) Gartner and Bukry, 1975 Amaurolithus delicatus Gartner and Bukry, 1975

Amaurolithus primus (Bukry and Percival, 1971) Gartner and Bukry, 1975

Amaurolithus tricorniculatus (Gartner, 1967) Gartner and Bukry, 1975

Calcidiscus leptoporus (Murray and Blackman, 1898) Loeblich and Tappan, 1978

Calcidiscus macintyrei (Bukry and Bramlette, 1969) Loeblich and Tappan, 1978

Calcidiscus premacintyrei Theodoridis, 1984

Catinaster calyculus Martini and Bramlette, 1963

Catinaster coalitus Martini and Bramlette, 1965

Coccolithus miopelagicus Bukry,1971

Coccolithus pelagicus (Wallich, 1877) Schiller, 1930

Coronocyclus nitescens (Kamptner, 1963) Bramlette and Wilcoxon, 1967

Cyclicargolithus floridanus (Roth and Hay in Hay et al., 1967) Bukry, 1971

Discoaster bellus Bukry and Percival, 1971

Discoaster berggrenii Bukry, 1971

Discoaster bollii Martini and Bramlette, 1963

Discoaster braarudii Bukry, 1971

Discoaster brouweri Tan (1927) emend. Bramlette and Riedel, 1954

Discoaster calcaris Gartner, 1967

Discoaster exilis Martini and Bramlette, 1963

Discoaster hamatus Martini and Bramlette, 1963

Discoaster kugleri Martini and Bramlette, 1963

Discoaster loeblichii Bukry, 1971

Discoaster misconceptus Theodoridis, $1984=$ Discoaster pentaradiatus

Discoaster musicus Stradner, 1959

Discoaster neohamatus Bukry and Bramlette, 1969

Discoaster neorectus Bukry, 1971

Discoaster pentaradiatus Tan (1927) emend. Bramlette and Riedel, 1954

Discoaster prepentaradiatus Bukry and Percival, 1971

Discoaster quinqueramus Gartner, 1969

Discoaster sanmiguelensis Bukry, 1981 = Discoaster musicus

Discoaster signus Bukry, 1971

Discoaster surculus Martini and Bramlette, 1963

Discoaster variabilis Martini and Bramlette, 1963

Minylitha convallis Bukry, 1973

Orthorhabdus serratus Bramlette and Wilcoxon, 1967 = Triquetrorabdulus serratus

Reticulofenestra haqii Backman, 1978

Reticulofenestra minuta Roth, 1970

Reticulofenestra minutula (Gartner, 1967) Haq and Berggren, 1978

Reticulofenestra pseudoumbilicus (Gartner, 1967) Gartner, 1969

Sphenolithus abies Deflandre in Deflandre and Fert, 1954

Sphenolithus heteromorphus Deflandre, 1953

Sphenolithus moriformis (Brönnimann and Stradner, 1960) Bramlette and Wilcoxon, 1967

Triquetrorhabdulus rioensis Olafsson, 1989

Triquetrorhabdulus rugosus Bramlette and Wilcoxon, 1967

Triquetrorhabdulus serratus (Bramlette and Wilcoxon, 1967) Olafsson, 1989 